\title{
HOMOGENISED LIMIT ANALYSIS OF MASONRY WALLS. PART I: FAILURE SURFACES
}

\author{
Abbreviated Title : Homogenised limit analysis of masonry, failure surfaces. \\ G.MILANI ${ }^{1 *}$, P.B.LOURENÇO ${ }^{2}$, A.TRALLI ${ }^{1}$ \\ ${ }^{1}$ Faculty of Engineering, University of Ferrara, Via Saragat 1, 44100-Ferrara (IT) \\ ${ }^{2}$ Department of Civil Engineering, School of Engineering, University of Minho, Azurém, 4800-058 Guimarães (PT)
}

\begin{abstract}
A simple micro-mechanical model for the homogenised limit analysis of in-plane loaded masonry is proposed. Assuming brickwork under plane stress condition and adopting a polynomial expansion for the 2D stress field, a linear optimisation problem is derived on the elementary cell in order to recover the homogenised failure surface of the brickwork. Different models of higher accuracy are obtained by increasing the degree of the polynomial approximation. Several cases of technical interest are treated in detail, varying both the geometrical characteristics of the cell and the mechanical properties of the components. The model is validated through comparisons with experimental data and kinematic approaches; the comparison with experimental data has the aim to reproduce in a qualitative way the shape of the failure surface, since 3D effects and brittle behaviour of bricks can not be taken into consideration with the model proposed.

In an accompanying paper, the homogenised failure surfaces here obtained are implemented in a finite element limit analysis code and relevant structural examples are treated both with a lower and an upper bound approach.
\end{abstract}

Keywords: masonry, homogenisation, limit analysis, micro-mechanics, composite behaviour

\footnotetext{
* Corresponding Author. Dipartimento di Ingegneria - Università degli Studi di Ferrara - Via Saragat 1 - 44100 Ferrara - Italy. e-mail: gmilani@ing.unife.it. Phone: +39 0532 974911. Fax: +39 0532974870.
} 


\section{INTRODUCTION}

In recent years, there has been a steady interest in the mechanics of unreinforced masonry structures, with the aim to provide efficient tools for better understanding their complex behaviour. Mortar joints usually present a lower strength than masonry units, so explaining the existence of planes of weakness along which cracks propagate at failure. Therefore, two main approaches have been developed for the constitutive description of masonry, usually known in the technical literature as macro-modelling and micro-modelling.

The macro-modelling approach does not make any distinction between masonry units (bricks, blocks, stones, etc.) and joints, averaging the effect of mortar through the formulation of a fictitious continuous material. This approach has been widely pursued in the past (the classical no-tension material [1], for instance, belongs to this category), because it makes possible to employ the rough discretizations necessary for actual large scale structures. Nevertheless, it appears really difficult to take into account some distinctive aspects of masonry, such as anisotropy in the inelastic range and the post-peak softening behaviour, closely related to the constituent materials (mortar and units) and to its geometry (bond pattern, thickness of joints, etc.). With the aim to take into account the above aspects, an equivalent macro-model has been recently developed in [2], featuring orthotropic elastic-plastic behaviour with softening. Nevertheless, the mechanical properties required by the model are derived from experimental data and the results are limited to the conditions under which the data are obtained. This means that the introduction of new materials and/or the application of a well known material in different loading conditions might require a different set of costly experimental programs.

The alternative micro-modelling approach consists in representing separately mortar joints and units. In some cases, reasonable simplifications have been introduced, for example utilising zero- 
thickness interfaces for the joints [3] [4]. Nevertheless, a drawback of this approach is related to the necessity of modelling separately units and mortar, so limiting its applicability to small panels.

Despite the wide employment of the homogenisation theory for modelling masonry structures in the elastic field, only a few papers extend the formulation to the inelastic range. Recently, a suitable homogenised model for the limit analysis of masonry walls has been presented in [5], where the units are supposed infinitely resistant and the joints are interfaces of zero thickness with a frictional failure surface. Furthermore, in [6], a brittle damaging model has been developed, characterized by an elementary cell composed by units, mortar and a finite number of fractures on the interfaces. Finally, in [7] a finite element approach has been adopted to represent the non linear behaviour of the homogenised material, assuming either elasto-plastic or damaging constitutive laws for units and mortar. Nevertheless, such finite element approach requires a great computational effort, since the field problem has to be solved numerically for each loading step, in all Gauss points. Furthermore, this choice needs a comprehensive mechanical characterisation of the constituent materials and large time both for the construction of the finite element model and for performing the analyses themselves.

On the other hand, the classical linear elastic analysis, which is the most disseminated tool in civil engineering design, fails to give an idea of the structural behaviour near collapse. This is a key issue, especially for historical constructions, where the low tensile strength of mortar joints leads to a very limited linear domain.

For the above reasons, limit analysis combined with homogenisation technique seems a powerful structural analysis tool to produce reliable results at collapse. This approach requires only a reduced number of material parameters and allows to avoid independent modelling of units and mortar. In addition, it provides limit multipliers of loads, failure mechanisms and, at least on critical sections, 
the stress distribution at collapse. Such approach has been recently presented for fibrous material ([8]) and it has been shown that reliable results can be obtained using this procedure.

Here, a simple model for the homogenised limit analysis of in-plane loaded masonry structures is presented. The model assumes fully equilibrated stress fields in the elementary cell, assigning polynomial expressions for the stress tensor components in a finite number of sub-domains, and imposes the continuity of the stress vector on the interfaces. In the framework of limit analysis, such stress distribution represents a statically admissible field in the elementary cell [9], and leads to a linear optimisation problem.

The paper is organised as follows: in Section 2, a brief review of the homogenisation theory in the rigid-plastic case is reported, whereas in Section 3 the proposed model is discussed in detail. With the aim to assess the accuracy of the results obtained, a preliminary comparison in the elastic range between the model and a standard f.e. procedure is provided. In Section 4, the micro-mechanical model is applied for some cases of technical interest for the evaluation of the homogenised failure surfaces of masonry. Several cases are reported and validated both against experimental data available in literature and, where possible, against closed-form solutions recently presented.

\section{HOMOGENISATION IN THE RIGID-PLASTIC CASE}

Masonry is a composite material usually made of units bonded with mortar joints. In most cases of building practice, units and mortar are periodically arranged. Such periodicity gives the possibility to consider an entire wall $\Omega$ as the repetition of a representative element of volume $Y$ (REV or elementary cell) - see Figure 1. $Y$ contains all the information necessary for describing completely the macroscopic behaviour of $\Omega$. If a running bond pattern is considered, as shown in Figure 1 , it can be easily checked that the elementary cell is rectangular. 
For periodic arrangements of units and mortar, homogenisation techniques can be used both in the elastic and inelastic range, taking into account the micro-structure only at a cell level. This leads to a significant simplification of the numerical models adopted for studying entire walls, especially for the inelastic case.

The basic idea of the homogenisation procedure consists in introducing averaged quantities representing the macroscopic stress and strain tensors (respectively $\mathbf{E}$ and $\boldsymbol{\Sigma}$ ), as follows:

$$
\begin{aligned}
& \mathbf{E}=<\boldsymbol{\varepsilon}>=\frac{1}{A} \int_{Y} \boldsymbol{\varepsilon}(\mathbf{u}) d Y \\
& \boldsymbol{\Sigma}=<\boldsymbol{\sigma}>=\frac{1}{A} \int_{Y} \boldsymbol{\sigma} d Y,
\end{aligned}
$$

where A stands for the area of the elementary cell, $\boldsymbol{\varepsilon}$ and $\boldsymbol{\sigma}$ stand for the local quantities (stresses and strains respectively) and $<*>$ is the averaging operator.

Periodicity conditions are imposed on the stress field $\boldsymbol{\sigma}$ and the displacement field $\mathbf{u}$, given by:

$$
\left\{\begin{array}{l}
\mathbf{u}=\mathbf{E y}+\mathbf{u}^{\text {per }} \quad \mathbf{u}^{\text {per }} \text { on } \partial Y \\
\boldsymbol{\sigma n} \text { anti-periodic on } \partial Y
\end{array}\right.
$$

where $\mathbf{u}^{\text {per }}$ stands for a periodic displacement field.

Let $S^{\mathrm{m}}, S^{\mathrm{b}}$ and $S^{\text {hom }}$ denote respectively the strength domains of the mortar, of the units and of the homogenised macroscopic material. It has been shown by Suquet [9] that the $S^{\text {hom }}$ domain of the equivalent medium is defined in the space of the macroscopic stresses as follows [10]: 


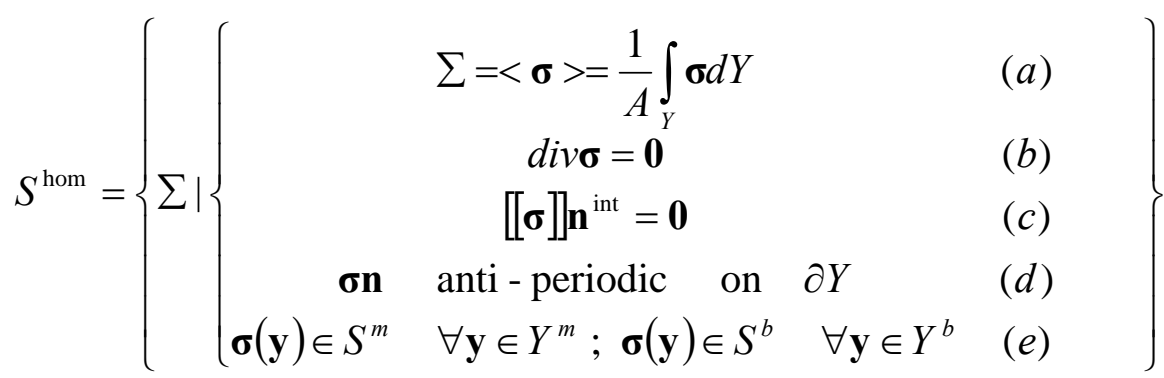

Here, $[[\boldsymbol{\sigma}]]$ is the jump of micro-stresses across any discontinuity surface of normal $\mathbf{n}^{\text {int }}$. Conditions (3-a) and (3-d) are derived from periodicity, condition (3-b) imposes the micro-equilibrium and condition (3-e) represents the yield criteria for the components (brick and mortar).

A dual kinematic definition of $S^{\text {hom }}$, also due to Suquet [9], can be derived through the support function $\pi^{\text {hom }}(\mathbf{D})$ as follows:

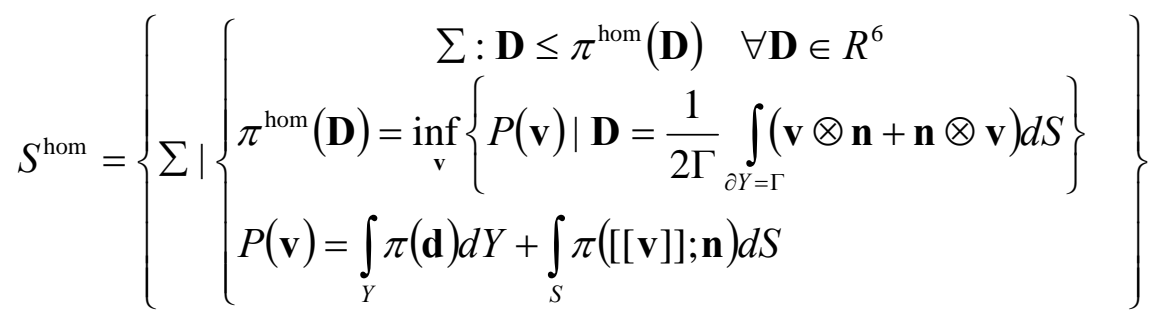

where:

- $\quad \mathbf{v}=\mathbf{D y}+\mathbf{v}^{\text {per }}$ is the microscopic velocity field

- $\quad \mathbf{v}^{\text {per }}$ is a periodic velocity field

- $\mathbf{d}$ and $\mathbf{D}$ are respectively the microscopic and macroscopic strain rate fields

- $S$ is any discontinuity surface of $\mathbf{v}$ in $Y, \mathbf{n}$ is the normal to $S$

- $\quad \pi([[\mathbf{v}]] ; \mathbf{n})=1 / 2([[\mathbf{v}]] \otimes \mathbf{n}+\mathbf{n} \otimes[[\mathbf{v}]])$

$-\pi(\mathbf{d})=\max _{\boldsymbol{\sigma}}\{\boldsymbol{\sigma}: \mathbf{d} ; \boldsymbol{\sigma} \in S(\mathbf{y})\}$ 
It is worth noting that, using the kinematic definition given by Eq. (4), it is possible to explicitly determine the homogenised strength domain of masonry in the space of the macroscopic stresses assuming infinitely resistant units and joints interfaces with Mohr-Coulomb failure criterion [5]. Alternatively, from the set of Eqs. (3), $S^{\text {hom }}$ can be statically obtained solving the following optimisation problem for every direction of the vector $\mathbf{n}_{\Sigma}$ :

$$
\left\{\begin{array}{c}
\max \{\hat{\lambda}\} \\
\text { such that }\left\{\begin{array}{c}
\hat{\lambda} \mathbf{n}_{\Sigma}=\hat{\lambda}\left[\begin{array}{lll}
\alpha_{11} & \alpha_{22} & \alpha_{12}
\end{array}\right]^{T}=\frac{1}{Y} \int_{Y} \boldsymbol{\sigma} d Y \\
\boldsymbol{\sigma n} \quad \text { anti - periodic on } \partial Y \\
\operatorname{div} \boldsymbol{\sigma}=\mathbf{0}
\end{array}\right. \\
\boldsymbol{\sigma}(\mathbf{y}) \in S^{i}(\mathbf{y})=\left\{\begin{array}{l}
S^{b} \text { if } \mathrm{y} \in \text { blocks } \\
S^{m} \text { if } \mathrm{y} \in \text { mortar }
\end{array}\right.
\end{array}\right.
$$

where:

- $\mathbf{n}_{\Sigma}=\left[\begin{array}{lll}\alpha_{11} & \alpha_{22} & \alpha_{12}\end{array}\right]^{T}$ is a versor in the macroscopic stress space $\Sigma_{11} \quad \Sigma_{22} \quad \Sigma_{12}$ - $\hat{\lambda} \mathbf{n}_{\Sigma}$ represents a macroscopic stress state on $S^{\text {hom }}$ belonging to a straight line from the origin of direction $\mathbf{n}_{\Sigma}$.

\section{A SIMPLE MICRO-MECHANICAL MODEL FOR DERIVING THE HOMOGENISED FAILURE SURFACE OF MASONRY}

In this Section a simple and numerically suitable approach for solving the optimisation problem given by Eqs. (5) is presented. As shown in Figure 2-a, one-fourth of the REV is sub-divided into nine geometrical elementary entities (sub-domains), so that all the cell is sub-divided into 36 subdomains, as shown in Figure 2-b. The subdivision adopted is the coarser (for $1 / 4$ of the cell) that can be obtained using rectangular geometries for every sub-domain. 
The macroscopic behaviour of masonry strongly depends on the mechanical and geometrical characteristics both of units and vertical/horizontal joints. For this reason, the subdivision adopted seems to be also particularly attractive, giving the possibility to characterise separately every component inside the elementary cell.

For each sub-domain, polynomial distributions of degree $(m)$ are a priori assumed for the stress components. Since stresses are polynomial expressions, the generic $i j^{\text {th }}$ component can be written as follows:

$$
\sigma_{i j}^{(k)}=\mathbf{X}(\mathbf{y}) \mathbf{S}_{i j}^{T} \quad \mathbf{y} \in Y^{k}
$$

where:

$-\quad \mathbf{X}(\mathbf{y})=\left[\begin{array}{lllllll}1 & y_{1} & y_{2} & y_{1}^{2} & y_{1} y_{2} & y_{2}^{2} & \ldots\end{array}\right] ;$

- $\quad \mathbf{S}_{i j}=\left[\begin{array}{lllllll}S_{i j}^{(1)} & S_{i j}^{(2)} & S_{i j}^{(3)} & S_{i j}^{(4)} & S_{i j}^{(5)} & S_{i j}^{(6)} & \ldots\end{array}\right] \quad$ is $\quad$ a $\quad$ vector $\quad$ of $\quad$ length $\quad(\tilde{N})$ $\left(\tilde{N}=\frac{m^{2}}{2}+\frac{3 m}{2}+1=\frac{(m+1)(m+2)}{2}\right)$ representing the unknown stress parameters;

- $\quad Y^{k}$ represents the $k^{\text {th }}$ sub-domain.

\subsection{EQUILIBRIUM AND ANTI-PERIODICITY OF $\sigma$ N ON $\partial V$}

The imposition of equilibrium (with zero body forces, as usually considered in homogenisation procedures) inside every sub-domain, the continuity of the stress vector on interfaces and antiperiodicity of $\boldsymbol{\sigma n}$ permit to strongly reduce the total number of independent stress parameters. More in detail, the imposition of micro-equilibrium $\left(\sigma_{i j, j}=0 \quad i=1,2\right)$ for every sub-domain yields: 


$$
\sum_{j=1}^{2} \mathbf{X}(\mathbf{y})_{, j} \mathbf{S}_{i j}^{(k) T}=\mathbf{0}
$$

Taking into consideration, for instance, the $2^{\text {nd }}$ order approximation the following equations can be written for the $k^{\text {th }}$ sub-domain:

$$
\left\{\begin{array}{l}
S_{11}^{(2 k)}+2 y_{1} S_{11}^{(4 k)}+y_{2} S_{11}^{(5 k)}+S_{12}^{(3 k)}+y_{1} S_{12}^{(5 k)}+2 y_{2} S_{12}^{(6 k)}=0 \\
S_{12}^{(2 k)}+2 y_{1} S_{12}^{(4 k)}+y_{2} S_{12}^{(5 k)}+S_{22}^{(3 k)}+y_{1} S_{22}^{(5 k)}+2 y_{2} S_{22}^{(6 k)}=0
\end{array}\right.
$$

As shown explicitly by Eq. (7b), Eq. (7a) represents a system of two equations in (2m) variables, each one being a polynomial expression of degree $(m-1)$ in $\boldsymbol{y}$.

A statically admissible stress distribution is obtainable if equilibrium is imposed everywhere inside each sub-domain, i.e. if $\sigma_{i j, j}(x, y)=0 \quad i=1,2 \quad \forall(x, y) \in$ sub-domain; since $\sigma_{i j}(x, y)$ is a polynomial expression of degree $(m)$, a linear combination of its derivatives $(\operatorname{div} \boldsymbol{\sigma})$ is a polynomial of degree $(m-1)$. Therefore Eq. (7) leads to write $(2 N)$ linear independent equations in the stress coefficients, where $N=\frac{(m-1)^{2}}{2}+\frac{3(m-1)}{2}+1=\frac{m(m+1)}{2}$. This condition permits the reduction, independently inside each sub-domain, of the total ( $3 \tilde{N})$ stress parameters to $(3 \tilde{N}-2 N)$ (with $3 \tilde{N}-2 N=\frac{(m+1)(m+6)}{2}$ ) unknowns.

It can be shown that each polynomial stress distribution can be rewritten in every sub-domain as follows:

$\sigma_{i j}^{(k)}=\hat{\mathbf{X}}_{i j}(\mathbf{y}) \hat{\mathbf{S}}^{(k)} \quad i=1,2 \quad k=1, \ldots, k^{\max } \quad \mathbf{y} \in Y^{k}$

where: 
- $\quad \hat{\mathbf{S}}^{(k)}$ is the vector of length $(3 \tilde{N}-2 N)$ of the remaining (linear independent) stress parameters of each sub-domain;

$-k^{\max }=9$.

A further reduction of the total $k^{\max }(3 \tilde{N}-2 N)$ unknowns is obtained a priori imposing the continuity of the stress vector on internal interfaces $\left(\sigma_{i j}^{(k)} n_{j}+\sigma_{i j}^{(r)} n_{j}=0 \quad i=1,2\right)$ for every $(k)$ and (r) contiguous sub-domains with a common interface of normal $\mathbf{n}$ (Figure 3).

Other two equations in the stress coefficients can be written for each interface as follows:

$\left(\hat{\mathbf{X}}_{i j}^{(k)}(\mathbf{y}) \hat{\mathbf{S}}^{(k)}+\hat{\mathbf{X}}_{i j}^{(r)}(\mathbf{y}) \hat{\mathbf{S}}^{(r)}\right) n_{j}=0 \quad i=1,2$

For example, if the vertical interface of Figure 3-b is considered, the following equations can be written for a quadratic interpolation of the stresses:

$\left\{\begin{array}{l}\sigma_{11}^{(k)}=\sigma_{11}^{(r)} \\ \sigma_{12}^{(k)}=\sigma_{12}^{(r)} \\ \text { with : } \\ \sigma_{11}^{(k)}=S_{11}^{(1 k)}+\left(y_{1}^{O(k)}-L^{(k)}\right) S_{11}^{(2 k)}+y_{2} S_{11}^{(3 k)}+\left(y_{1}^{O(k)}-L^{(k)}\right)^{2} S_{11}^{(4 k)}+y_{2}\left(y_{1}^{O(k)}-L^{(k)}\right) S_{11}^{(5 k)}+y_{2}^{2} S_{11}^{(6 k)} \\ \sigma_{11}^{(r)}=S_{11}^{(1 r)}+y_{1}^{O(r)} S_{11}^{(2 r)}+y_{2} S_{11}^{(3 r)}+\left(y_{1}^{O(r)}\right)^{2} S_{11}^{(4 r)}+y_{2} y_{1}^{O(r)} S_{11}^{(5 r)}+y_{2}^{2} S_{11}^{(6 r)} \\ \sigma_{12}^{(k)}=S_{12}^{(1 k)}+\left(y_{1}^{O(k)}-L^{(k)}\right) S_{12}^{(2 k)}+y_{2} S_{12}^{(3 k)}+\left(y_{1}^{O(k)}-L^{(k)}\right)^{2} S_{12}^{(4 k)}+y_{2}\left(y_{1}^{O(k)}-L^{(k)}\right) S_{12}^{(5 k)}+y_{2}^{2} S_{12}^{(6 k)} \\ \sigma_{12}^{(r)}=S_{12}^{(1 r)}+y_{1}^{O(r)} S_{12}^{(2 r)}+y_{2} S_{12}^{(3 r)}+\left(y_{1}^{O(r)}\right)^{2} S_{12}^{(4 r)}+y_{2} y_{1}^{O(r)} S_{12}^{(5 r)}+y_{2}^{2} S_{12}^{(6 r)}\end{array}\right.$

where:

- $y_{1}^{O(k)}$ is the origin abscissa of the frame of reference belonging to the $k^{\text {th }}$ sub-domain (Figure 3-a) in the local frame of reference $y_{1}-y_{2}$

- $y_{1}^{O(r)}=y_{1}^{O(k)}-L^{(k)}$

- $L^{(k)}$ length of the $k^{\text {th }}$ sub-domain. 
A statically admissible stress distribution can be obtained if continuity of the stress vector is guaranteed in any point of the interface. Being Eqs. (9) polynomial expressions of degree $(m)$ in the abscissa $s$ of the interface, other $2 N^{\prime}$ equations (where $N^{\prime}=m+1$ ) in $\hat{\mathbf{S}}^{(k)}$ and $\hat{\mathbf{S}}^{(r)}$ for each $(k \leftrightarrow r)$ interface can be written from (9), see Figure 3-b.

Eq. (9) involves variables belonging to contiguous sub-domains, while Eq. (7) involves only the stress parameters of a single sub-domain.

Finally anti-periodicity of $\boldsymbol{\sigma n}$ on $\partial V$ requires $2 N^{\prime}$ additional equations per pair of external faces (m) (n) (Figure 3-c), i.e. it should be imposed that stress vectors $\boldsymbol{\sigma} \mathbf{n}$ are opposite on opposite sides of $\partial V$ :

$\hat{\mathbf{X}}_{i j}^{(m)}(\mathbf{y}) \hat{\mathbf{S}}^{(m)} \mathbf{n}_{1}=-\hat{\mathbf{X}}_{i j}^{(n)}(\mathbf{y}) \hat{\mathbf{S}}^{(n)} \mathbf{n}_{2}$

Where $\mathbf{n}^{(m)}$ and $\mathbf{n}^{(n)}$ are oriented versors of the external faces of the paired sub-domains $(m)(n)$. As it is possible to note, Eq. (9c) are similar to the internal interfaces equilibrium equations (9a), but in this case the stress anti-periodicity requires $p_{a p} 2 N^{\prime}$ additional equations, where $p_{a p}=12$ is the total number of external sub-domains pairs.

Some elementary assemblage operations on the local variables (handled automatically) lead to write the stress vector inside every sub-domain as follows:

$\tilde{\boldsymbol{\sigma}}^{(k)}=\tilde{\mathbf{X}}^{(k)}(\mathbf{y}) \tilde{\mathbf{S}} \quad k=1, \ldots, k^{\max }$

where:

- $\quad \tilde{\boldsymbol{\sigma}}^{(k)}$ is the vector of membrane actions inside the $k^{\text {th }}$ sub domain;

- $\quad \tilde{\mathbf{X}}^{(k)}(\mathbf{y})$ is a $3 \mathrm{x} N_{u n}$ matrix which contains only geometrical coefficients; its elements are polynomial forms in the microscopic coordinate $\mathbf{y}$;

- $\quad \tilde{\mathbf{S}}$ is the vector (of length $N_{u n}$ ) of the total stress parameters unknown. 
For the examples reported here, all the equations necessary to ensure equilibrium and antiperiodicity have been handled symbolically by means of the Symbolic Math Toolbox ${ }^{\mathrm{TM}}$ and all the procedure described has been implemented in Matlab ${ }^{\mathrm{TM}}$ language (V.6.1-R.12.1) on MSWin Platform [11]. Optimisation has been treated modifying some routines in the Optimisation Math Toolbox $^{\mathrm{TM}}[12]$.

The equations written in order to satisfy internal equilibrium, equilibrium on interfaces and antiperiodicity of the stress vector lead to system of equations $\mathbf{A S}=\mathbf{0}$, where $\mathbf{S}$ is the vector of total stress parameters. Nevertheless, not all the rows of this system are linearly independent. This can be easily shown if four generic rectangular elements with four common interfaces and subjected only to constant non zero shear stress are considered, as reported in Figure 3-d. Internal equilibrium is apriori satisfied, whereas four equations for ensuring equilibrium on interfaces have to be written. Nevertheless, only three of these four equations are linear independent.

The linear dependence of some equations with respect to others has been handled automatically by means of the Symbolic Math Toolbox ${ }^{\mathrm{TM}}$, checking the rank of matrix $\mathbf{A}$ and progressively eliminating linearly dependent rows.

Finally, four different models of increasing accuracy $\left(\mathrm{P}_{0} \mathrm{P}_{2} \mathrm{P}_{3} \mathrm{P}_{4}\right)$ have been obtained increasing the degree of the polynomial expansion.

\subsection{LINEAR ELASTIC CASE}

A preliminary study in the linear elastic range has been carried out with the aim to test the reliability of the results obtained using the micro mechanical model.

Let us consider the quadratic functional $\Pi$ of the complementary energy evaluated in the REV. With the assumption done in Eq. (10), an approximation of $\Pi$ can be written as follows: 


$$
\Pi^{*}=\sum_{k=1}^{k^{\max }} \int_{Y_{k}} \frac{1}{2} \widetilde{\mathbf{S}}^{T} \widetilde{\mathbf{X}}^{(k) T}(\mathbf{y}) \mathbf{C}^{b, m} \tilde{\mathbf{X}}^{(k)}(\mathbf{y}) \widetilde{\mathbf{S}} d Y_{k}-\sum_{j} \int_{S_{j}} \widetilde{\mathbf{S}}^{T} \widetilde{\mathbf{X}}^{(k) T} \overline{\mathbf{u}} d S_{j}
$$

Where $\mathbf{C}^{b, m}$ is the compliance matrix of units or mortar joints and $\overline{\mathbf{u}}$ is the displacement imposed on the boundary $\partial Y$ of the elementary cell, representing a given macroscopic strain tensor $\mathbf{E}$.

The minimization of the approximated expression of $\Pi$ leads to the following expression:

$$
\begin{aligned}
& \nabla \Pi^{*}=\left(\sum_{k=1}^{k^{\max }} \int_{Y_{k}} \tilde{\mathbf{X}}^{(k) T}(\mathbf{y}) \mathbf{C}^{b, m} \tilde{\mathbf{X}}^{(k)}(\mathbf{y}) d Y_{k}\right) \tilde{\mathbf{S}}-\sum_{j} \int_{S_{j}} \tilde{\mathbf{X}}^{(k) T}(\mathbf{y}) \overline{\mathbf{u}} d S_{j}= \\
& =\mathbf{C}^{\mathrm{hom}} \widetilde{\mathbf{S}}-\overline{\mathbf{U}}=0
\end{aligned}
$$

which enables to find both $\widetilde{\mathbf{S}}$, by factorization of the matrix $\mathbf{C}^{\text {hom }}$ and $\boldsymbol{\Sigma}$, from integration of the local stress field, see Eq. (1).

In this Section, a comparison between the elastic moduli provided by the model proposed and a standard numerical 2D FEM procedure ([13]) is presented for a case of practical interest. The dimensions of the units are assumed to be 250x120x55 mm (brick UNI5628/65) and the thickness of mortar joints is equal to $10 \mathrm{~mm}$. The initial mechanical characteristics of materials are reported in Table I; the simulations are handled keeping the brick Young modulus $\mathrm{E}_{\mathrm{b}}$ constant and progressively reducing the mortar Young modulus $E_{m}$, so assuming a wide range of $E_{b} / E_{m}$ ratios (from 5 to 90), in order to simulate also historical brickwork. In Figure 4, a comparison between the homogenised $A_{\mathrm{ijhk}}$ membrane moduli obtained is reported varying $\mathrm{E}_{\mathrm{b}} / \mathrm{E}_{\mathrm{m}}$ ratio. The homogenised moduli are normalised versus the corresponding moduli of the brick. As it can be noticed, the provided moduli are reliable in a large range of $E_{b} / E_{m}$ ratios, even for the most simple model with constant stresses tensor (P0). Nevertheless, Figure 4-a shows that a progressively reduced accuracy of the P0 model can be noticed for the $A_{1111}$ module, due to the presence of shear stresses in the bed joint (as already discussed in [14]). Finally, in Figure 5 the relative error, in terms of energy norm, 
arising from a comparison with F.E. results is reported. It can be observed that models P3 and P4 exhibit very satisfactory accuracy also for very low values of mortar Young modulus.

\subsection{ADMISSIBLE STRESS FIELDS}

The approximation of the stress field automatically built up represents a statically admissible stress field, therefore the static theorem of limit analysis provides the following optimisation problem:

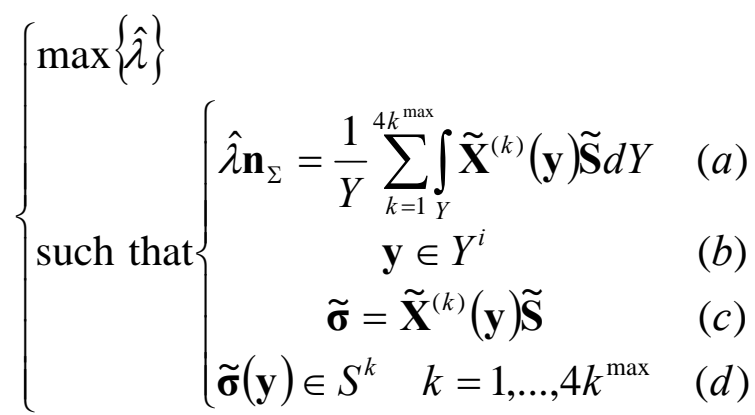

where $S^{i}$ stands for the failure surface of the component (unit or mortar) belonging to the $i^{\text {th }}$ subdomain.

The optimisation problem given by Eqs. (13) is generally non-linear [15], as a consequence of the (possible) non-linearity of the strength functions of the components. In addition, condition (13-d) has to be checked in every point of the domain $Y$. Nevertheless, as suggested in a classical paper by Belytschko and Hodge [16], the check could be avoided imposing the material admissibility only where the stress status is the maximum. This is feasible only for the P0 and P1 models; alternatively, the discretisation proposed here consists in enforcing, in every sub-domain, the admissibility condition in a regular grid of "nodal points" with step rxq.

Adopting such regular grid, the optimisation problem reduces to the following discretised form: 


$$
\left\{\begin{array}{l}
\max \{\hat{\lambda}\} \\
\text { such that }\left\{\begin{array}{c}
\hat{\lambda}_{\mathbf{n}_{\Sigma}}=\frac{1}{Y} \sum_{k} \int_{Y} \tilde{\mathbf{X}}^{(k)}(\mathbf{y}) \tilde{\mathbf{S}} d Y \\
\mathbf{y}^{j} \equiv \text { nodal point } \\
\tilde{\boldsymbol{\sigma}}^{j}=\tilde{\mathbf{X}}^{(k)}\left(\mathbf{y}^{j}\right) \widetilde{\mathbf{S}} \\
\tilde{\boldsymbol{\sigma}}^{j} \in S^{i} \quad j=1, \ldots, r q \\
k=1, \ldots, 4 k^{\max }
\end{array}\right.
\end{array}\right.
$$

Optimisation problem given by Eqs. (14) generally remains non-linear. In order to use linear programming algorithms, each of the non-linear inequalities of Eqs. (14) could be approximated by a set of linear inequalities (as proposed in the past, for instance, by Anderheggen and Knopfel [17] or Maier [18]), by replacing the yield surfaces with inscribed hyper polyhedrons. Finally, the convergence of the solution obtained should be checked progressively increasing the number of planes of the approximation, as recently pointed out, see Sloan and Kleeman ([19]) and Olsen ([20]).

Alternatively and more efficiently, an iterative procedure is adopted here, taking advantage of the fact that the simplex method proceeds from basic solution to basic solution towards an optimal basic solution, i.e. on the vertices of the hyper polyhedron ([21]).

It is stressed that the simplex method can rarely compete favourably with the more recent and efficient interior-point methods (IPMs), see e.g. Mehrotra [22]. In particular, Klee and Minty [23] have proven that the worst-case complexity of some variants of the simplex method is exponential, while interior-point methods have only polynomial complexity. Nevertheless, for the present application with a very limited number of variables and inequality constraints of the problem, simplex-type algorithms are still preferable than IPMs.

The basic idea of the iterative procedure adopted is the following: in the starting step, a coarse linear approximation of the non linear failure surfaces of the components is adopted, as shown in 
Figure 6-a. The application of the simplex method in the optimisation at the $i^{\text {th }}$ step leads to an optimal solution in a corner of the domain. From the iterative $i^{\text {th }}$ solution point a new tangent plane is added in $\mathrm{P}^{\prime}$, as shown in Figure 6-b, so restarting an $(i+1)^{\text {th }}$ optimisation procedure. The iterations continue until a fixed tolerance in the error between the $i^{\text {th }}$ and $(i+1)^{\text {th }}$ solution is reached.

The aim of the procedure is to give a precise approximation of the yield surface only near the solution of the problem. The described algorithm leads to a well approximated (even if non rigorous) lower bound, being the yielding surface approximated with a circumscribed polyhedron.

Figure 7 represents the convergence of the multiplier upon refining the grid of the nodal points where the material admissibility is checked. It can be observed that a coarse grid is sufficient to obtain adequate results. Therefore, a regular grid of 5x5 nodal points will be generally employed in the subsequent part of the paper.

In Figure 8, the strength domain obtained increasing the degree of the polynomial expansion is represented in the macroscopic stress space with $\Sigma_{12}=0$; the results are compared with a full finite element limit analysis on the REV. Mechanical characteristics of the constituent materials are summarized in Table II; a Mohr-Coulomb failure criterion in plane stress is chosen for mortar joints, while units are supposed infinitely resistant; the geometry of the elementary cell is reported in Table III.

As Figure 8 shows, the model with constant stress tensor (P0) is unable to reproduce the typical anisotropic behaviour of masonry at failure ([24]), while the refined models give a progressively increased accuracy of the results (especially P3 and P4) in comparison with the f.e. analysis. Therefore, the cubic interpolation P3 will be generally employed in the subsequent part of the Paper. 


\section{VALIDATION AND DISCUSSION OF HOMOGENISED FAILURE SURFACES}

In this Section, some meaningful cases are discussed, with the aim to test the reliability of the results obtained. In the first example, a comparison between the failure surfaces obtained by de Buhan and de Felice ([5]) and those derived using the present model is discussed. The example is meaningful because it tests the reliability of the model proposed in the tension-tension range in comparison with a closed-form solution obtained through a kinematic approach. In the second example, the micro-mechanical model is applied to obtain homogenised failure surfaces for the masonry material considered by Page ([25]) in his tests on half-scale panels.

Moreover, the homogenised surfaces for the masonry experimentally tested by Raijmakers and Vermeltfoort ([26]) are provided supposing different mechanical properties for the constituent materials. In addition, for the latter example, a kinematic approach is adopted, supposing both the units infinitely resistant and the joints interfaces with a frictional type failure surface with cap in compression. Finally, a comparison between the static and kinematic approach is reported in the compression-compression range.

\subsection{COMPARISON WITH AN EXPLICIT SOLUTION IN THE TENSION-TENSION REGIME}

An explicit homogenised strength domain in the case of infinitely resistant units and joints reduced to interfaces has been derived in [5] by de Buhan and de Felice. The reduction of joints to interfaces with a Mohr-Coulomb failure criterion combined with the infinitely resistance of units, makes possible to find, through a kinematic approach, an explicit solution for the homogenisation problem in the rigid-plastic case. It can be shown that the homogenised material so derived is infinitely 
resistant in the compression-compression region, while is orthotropic at failure in the tensiontension field.

The numerical failure surfaces presented in this Section (and in what follows) are obtained solving the optimisation problem given by Eqs. (14), where the direction of the load $\left[\begin{array}{lll}\Sigma_{11} & \Sigma_{22} & \Sigma_{12}\end{array}\right]^{T}$ depends on the orientation $\vartheta$ of the principal directions with respect to the material axes (Figure 9):

$$
\left\{\begin{array}{c}
\max \{\hat{\lambda}\} \\
\operatorname{such} \text { that }\left\{\begin{array}{c}
\hat{\lambda} \mathbf{n}_{\Sigma}=\frac{1}{Y} \sum_{k} \int_{Y} \tilde{\mathbf{X}}^{(k)}(\mathbf{y}) \tilde{\mathbf{S}} d Y \\
\mathbf{y}^{j} \equiv \text { nodal point } \\
\tilde{\boldsymbol{\sigma}}^{j}=\tilde{\mathbf{X}}^{(k)}\left(\mathbf{y}^{j}\right) \tilde{\mathbf{S}} \\
n_{\Sigma, 1}=\frac{1}{2}(\cos (\psi)(1+\cos (2 \vartheta))+\sin (\psi)(1-\cos (2 \vartheta))) \\
\tilde{\boldsymbol{\sigma}}^{j} \in S^{k} \quad \begin{array}{c}
j=1, \ldots, r q \quad k=1, \ldots, 4 k^{\max } \\
n_{\Sigma, 2}=
\end{array} \\
n_{\Sigma, 3}=\frac{1}{2}(\cos (\psi)(1-\cos (2 \vartheta))+\sin (\psi)(1+\cos (2 \vartheta)))
\end{array}\right.
\end{array}\right.
$$

where:

- $\psi$ denotes the loading angle, given by $\tan (\psi)=\frac{\Sigma_{v}}{\Sigma_{h}}$

- $\vartheta$ denotes the angle between $\Sigma_{h}$ and the $x$-axis.

In Figure 10, a comparison between [5] and the failure surfaces obtained using the proposed model with joints lumped to interfaces is represented. A Mohr-Coulomb failure criterion is used for mortar joints; three different orientations $\vartheta$ are inspected. The material properties and geometry of the elementary cell are the same as in Section 3, see Table II and Table III respectively.

Closed-form results are obviously well reproduced by the model adopting an interface law for the joints (Figure 10), whereas remarkable differences in the shape of the surface are present taking into 
accont the actual thickness of the joint $(10 \mathrm{~mm})$ and adopting, for instance, for the mortar a MohrCoulomb failure criterion in plane-stress (Figure 11).

The dependence of the homogenised failure surfaces both on the mechanical properties of components and on the geometry of the elementary cell (dimensions of units, thickness of joints) demonstrates that the micro-mechanical model proposed is a valuable general tool for the analysis at collapse of masonry.

\subsection{COMPARISON WITH EXPERIMENTAL DATA}

The most complete set of strength data of masonry under biaxial loads has been provided by Page [25], who tested 102 panels of half-scale solid clay brick masonry with dimensions 360x360x50 $\mathrm{mm}^{3}$. The dimensions of the units were $110 \times 50 \times 35 \mathrm{~mm}^{3}$ [27] and the mortar thickness was $10 \mathrm{~mm}$. The panels were loaded proportionally in the principal stress directions $\Sigma_{h}$ and $\Sigma_{v}$ along different orientations $\vartheta$ with respect to the material axes, see Figure 9.

Material properties for mortar and units are given in Table IV. Both for mortar joints and units, a Mohr-Coulomb failure criterion in plane stress is adopted. It has to be emphasized that the results reported in [25] and [28] provide only the mean compressive strenght of mortar ( $\cong 5 \mathrm{MPa}$ ) and bricks (=15.41 MPa), insufficient for a full parametric identification of the model in plane stress. Furthermore, it should be underlined that the model at hand is only capable of reproducing the shape of the failure surface and not the actual strength in compression from the masonry components, since 3D effects are neglected and, in the framework of limit analysis, a ductile behaviour of the bricks is assumed. For these reasons, mechanical characteristics of constituent materials are assumed with the aim of fitting experimental data reported in [28] and [29]. 
In Figure 12-a the homogenised failure surface for the orientation $\vartheta=0^{\circ}$ is reported in comparison with experimental data by Page. Furthermore, in Figure 12-b and Figure 12-c failure surfaces numerically obtained and the experimental data are compared for the orientations $\vartheta=22.5^{\circ}$ and $\vartheta=45^{\circ}$, which are in close agreeement.

\subsection{COMPARISON WITH AN EXPLICIT SOLUTION IN THE COMPRESSION- COMPRESSION RANGE}

The brickwork considered by Raijmakers and Vermeltfoort ([26]) for performing some experimental tests on shear walls is here examined. The units dimensions are $210 \times 52 \times 100 \mathrm{~mm}^{3}$, whereas the thickness of the mortar joints is $10 \mathrm{~mm}$. Such shear walls have been already examined through numerical simulations and a micro-mechanical approach by Lourenço and Rots [3], [29] and Sutcliffe et al. [30]. In their analyses, both authors reduce the joints to interfaces and adopt for mortar a frictional-type failure behaviour. A non linear cap model is chosen in [3], whereas a linear approximation of the cap, as shown in Figure 13, is adopted in [30], with the aim to study the shear walls in the framework of limit analysis.

In the present paper, three different models (A, B, C) for joints are critically examined (Table V); in model $\mathrm{A}$ and $\mathrm{B}$ joints are reduced to interfaces, whereas in model $\mathrm{C}$ the actual thickness of the mortar is considered, adopting a Mohr-Coulomb failure criterion in plane stress. Model A and B differ only for the compressive cap, which is vertical in model A and with a very prominent shape in model B; furthermore, the value of cohesion adopted for model C is deduced supposing the same uniaxial compressive strength of the cases A and B.

The goal of the comparison is to evaluate both the accuracy of the static approach in comparison with kinematic procedures and the differences in the homogenised failure surfaces in presence of 
different mechanical characteristics for mortar (models A and B) and actual thickness of the joints (model C).

The homogenised surfaces in the compression-compression region obtained using the three different models illustrated are reported in Figure 14-a $\left(\vartheta=0^{\circ}\right)$, Figure 14 -b $\left(\vartheta=22.5^{\circ}\right)$ and Figure 14-c $\left(\vartheta=45^{\circ}\right)$. As it can be noticed, the resultant failure surfaces are strongly dependent on the mechanical characteristics adopted for the joints. Furthermore, the shape adopted for the compressive cap in Model B strongly affects its ultimate strength in the compression region. The results previously discussed show that the homogenised surface depends on the geometrical and mechanical characteristics assumed for the components and that the proposed model is able to reproduce in a very simple manner the macroscopic strength domain whenever different failure behaviours for the components are taken into account.

It is worth noting that a comparison with a kinematic formulation is possible for joints reduced to interfaces (i.e. for models A and B). In fact, the kinematic formulation of limit analysis leads to the following constrained minimisation problem:

$$
\left\{\begin{array}{c}
\chi=\min _{\mathbf{v}^{p e r}, \mathbf{D}} \frac{1}{A} \int_{A} P(\mathbf{d}) d S \\
\sum^{0}: \mathbf{D}=1 \\
\mathbf{v}=\mathbf{D y}+\mathbf{v}^{\text {per }}
\end{array}\right.
$$

where $\chi$ is the kinematic limit multiplier of the assigned macroscopic stress $\Sigma^{0}$ and $P(\mathbf{d})$ is the local plastic dissipation over the REV. Optimisation problem given by Eqs. (16) has been treated in detail by some authors in the past for obtaining the homogenised failure surfaces for composites materials (see e.g. [8]). In the case of mortar joints reduced to interfaces with a linearized frictionaltype failure criterion, as shown in Figure 13, and for units infinitely resistant, the plastic dissipation depends only on the jump of displacements on interfaces and Eqs. (14) can be re-written as follows: 


$$
\left\{\begin{array}{c}
\chi=\min _{\mathbf{v}} \frac{1}{\Gamma} \int_{\Gamma}[[\mathbf{v}]] \boldsymbol{\sigma} d s \\
\sum^{0}: \mathbf{D}=1 \\
{[[\mathbf{v}]]=\sum_{i=1}^{n} \dot{\lambda}_{i} \nabla_{\boldsymbol{\sigma}} f^{(i)}}
\end{array}\right.
$$

where $\dot{\lambda}_{i}$ is the plastic multiplier associated with the (linear) inequality constraint $f^{(i)} \leq 0$ which determines the admissible stress state. The optimisation problem given by Eqs. (17) can be easily handled numerically and leads to reproduce $S^{\text {hom }}$ through a kinematic approach.

For models A and B, a comparison between the model proposed and the kinematic approach is illustrated respectively in Figure 15 and Figure 16. The comparison between the homogenized surfaces $S^{\text {hom }}$ obtained through the static approach and a kinematic procedure shows the accuracy of the proposed model.

\section{CONCLUSIONS}

In the present paper, a novel micro-mechanical model in plane stress for the homogenised limit analysis of masonry subjected to in-plane loading has been presented. Adopting a polynomial expansion for the stresses field, a simple linear optimisation problem has been derived on the elementary cell with the aim to find the homogenised failure surface of masonry. Four different models of increasing accuracy have been obtained progressively raising the degree of the polynomial approximation. The model presented is able to satisfactory reproduce homogenised failure surfaces of masonry varying both the geometrical characteristics of the cell and the mechanical properties of the components. The accuracy of the model has been assessed through meaningful comparisons both with kinematic approaches. The shape of experimental failure surfaces can also be reproduced with the model at hand. For this reason, it appears a simple and 
useful task to reproduce experimental data also when only compressive strength of masonry along few directions is known.

In an accompanying paper, the homogenised failure surfaces here recovered will be implemented in a f.e. limit analysis code and meaningful structural examples will be treated in detail both with a lower and an upper bound approach.

\section{ACKNOWLEDGEMENTS}

A.Tralli and G.Milani gratefully acknowledge the support of the research project MIUR COFIN 2003 - Interfacial damage failure in structural systems. Coordinator: Prof. A. Tralli.

\section{REFERENCES}

[1] Di Pasquale S. New trends in the analysis of masonry structures. Meccanica 1992; 27: 173-184.

[2] Lourenço PB, de Borst R, Rots JG. A plane stress softening plasticity model for orthotropic materials. International Journal for Numerical Methods in Engineering 1997; 40: 4033-4057.

[3] Lourenço PB, Rots J. A multi-surface interface model for the analysis of masonry structures. Journal of Engineering Mechanics ASCE 1997; 123 (7): 660-668.

[4] Lotfi HR, Shing PB. Interface model applied to fracture of masonry structures. Journal of Structural Engineering ASCE 1994; 120 (1): 63-80.

[5] de Buhan P, de Felice G. A homogenisation approach to the ultimate strength of brick masonry. Journal of the Mechanics and Physics of Solids 1997; 45 (7): 1085-1104.

[6] Luciano R, Sacco E. Homogenisation technique and damage model for old masonry material. International Journal of Solids and Structures 1997; 34 (24): 3191-3208.

[7] Pegon P, Anthoine A. Numerical strategies for solving continuum damage problems with softening: application to the homogenisation of masonry. Computers and Structures 1997; 64 (1-4): 623-642.

[8] Carvelli V, Maier G, Taliercio A. Kinematic limit analysis of periodic heterogeneous media. Comput. Mod. Engng. Sci. 2000; 1(2): 15-26. 
[9] Suquet P. Analyse limite et et homogeneisation. Comptes Rendus de l'Academie des Sciences - Series IIB Mechanics 1983; 296: 1355-1358.

[10] Taliercio A, Sagramoso P. Uniaxial strength of polymeric-matrix fibrous composites predicted through a homogenization approach. International Journal of Solids and Structures 1995; 32 (14): 2095-2123.

[11] Matlab User’s Guide, Version 6. The MathWorks, June 2001.

[12] Optimisation Toolbox User’s Guide, Version 2.1.1. The MathWorks, June 2001.

[13] Anthoine A. Derivation of the in-plane elastic characteristics of masonry through homogenisation theory. International Journal of Solids and Structures 1995; 32 (2): 137-163.

[14]Zucchini A, Lourenco PB. A micro-mechanical model for the homogenisation of masonry. International Journal of Solids and Structures 2002; 39: 3233-3255.

[15] Lyamin AV., Sloan SW. Lower bound limit analysis using nonlinear programming. International Journal for Numerical Methods in Engineering 2002; 55 (5): 573-611.

[16]Belytschko T, Hodge PG. Plane stress limit analysis by finite elements, ASCE Journal of the Engineering Mechanics Division December 1970; 931-943.

[17] Anderheggen E, Knopfel H. Finite element limit analysis using linear programming. . International Journal of Solids and Structures 1972; 8: 1413-1431.

[18] Maier G. Mathematical programming methods for deformation analysis at plastic collapse. Computers and Structures 1977; 7: 599-612.

[19] Sloan SW, Kleeman PW. Upper bound limit analysis using discontinuous velocity fields. Computer Methods in Applied Mechanics and Engineering 1995; 127 (1-4): 293-314.

[20] Olsen PC. The Influence of the linearisation of the yield surface on the load bearing capacity of reinforced concrete slabs. Computer Methods in Applied Mechanics and Engineering 1998; 162: 351-358.

[21]Cannarozzi AA, Sacchi PL, Tralli A. On the limit analysis of steel structures in presence of shear. Journal de Mecanique Theorique et Appliquee 1982 ; 1 (3) : 379-401.

[22] Mehrotra S. On the implementation of a (primal-dual) interior point method. SIAM Journal on Optimization 1992; 2(4): 575-601.

[23] Klee V, Minty G. How good is the simplex algorithm?. In: Shisha O, editor. Inequalities-III. New York: Academic Press, 159-175, 1972. 
[24] Page AW. A biaxial failure criterion for brick masonry in the tension-tension range. International Journal of Masonry Constructions 1981; 1: 26-30.

[25] Page AW. The biaxial compressive strength of brick masonry. Proc. Instn Civ. Engrs. 1981 ; Part 2, 71, Sept.: 893906.

[26] Raijmakers TMJ, Vermeltfoort A. Deformation controlled tests in masonry shear walls (in Dutch). Report B-921156, TNO-Bouw, Delft, The Netherlands, 1992.

[27] Andreaus U, Maroder M. Stato dell'arte sui legami costitutivi dei solidi murari (In Italian). Studi e Ricerche 2/91 Misc. RO-DS-SR (E), Università degli Studi di Roma La Sapienza, Dipartimento di Ingegneria Strutturale e Geotecnica, Rome, 1991.

[28] Dhanasekar M, Page AW, Kleeman PW. The failure of brick masonry under biaxial stresses. Proc. Instn. Civ. Engrs 1985; Part 2, 79, June : 295-313.

[29]Lourenço PB. Computational strategies for masonry structures. PhD Thesis 1996. Available at www.civil.uminho.pt/masonry.

[30] Sutcliffe DJ, Yu HS, Page AW. Lower bound limit analysis of unreinforced masonry shear walls. Computers and Structures 2001; 79: 1295-1312. 


\section{LIST OF FIGURES:}

Figure 1: Periodic structure $\left(\mathrm{X}_{1} \mathrm{X}_{2}\right.$ : macroscopic frame of reference) and elementary cell $\left(y_{1} y_{2}\right.$ : local frame of reference).

Figure 2: Subdivision in sub domains adopted: (-a) subdivision and geometrical characteristics of one-fourth of the elementary cell, (-b) subdivision into 36 sub-domains for the entire cell.

Figure 3: Contiguous sub-domains. -a: geometry and frame of reference of the sub-domains; -b: vertical/horizontal interfaces between adjacent sub-domains.

Figure 4: Homogenised in-plane moduli. -a: $A_{1111}$ modulus; -b: $A_{1212}$ modulus; -c: $A_{1122}$ modulus; $\mathrm{d}: A_{2222}$ modulus.

Figure 5: Relative error, in terms of energy norm, for models proposed versus with f.e. results.

Figure 6: Iterative procedure utilised in the optimisation problem.

Figure 7: Convergence of the limit multiplier upon refining the grid of points with material admissibility evaluation.

Figure 8: Failure surface in the tension-tension range for the models proposed without shear actions.

Figure 9: Biaxial tests on masonry for different orientations of the external load with respect to the bed joint.

Figure 10: Comparison between P4/P3 models and the explicit solution of de Buhan and de Felice [5] in the tension-tension range for different orientations $\vartheta$.

Figure 12: Homogenized failure surface for Page results [25] for P3 model. -a: $\vartheta=0^{\circ}$; -b: $\vartheta=22.5^{\circ} ;-\mathrm{c}: \vartheta=45^{\circ}$.

Figure 11: Failure surfaces in the tension-tension range obtained using P3 model, assuming infinitely resistant units and mortar joints with a Mohr-Coulomb failure criterion (solid line: joints 
reduced to interfaces; dashed line: plane stress condition). -a: directions $\vartheta=0^{\circ}, 90^{\circ}$; -b: directions $\vartheta=22.5^{\circ}, 67.5^{\circ}$; -c: directions $\vartheta=45^{\circ}$.

Figure 13: Interface failure surface adopted for mortar joints.

Figure 14: Homogenized failure surfaces assuming different mechanical characteristics for the joints (rigid blocks); -a: $\vartheta=0^{\circ}$; -b: $\vartheta=22.5^{\circ} ;$-c: $\vartheta=45^{\circ}$.

Figure 15: Comparison between a kinematic approach and the micro-mechanical model presented for model A (-a: $\vartheta=0^{\circ}$; -b: $\vartheta=22.5^{\circ}$; -c: $\left.\vartheta=45^{\circ}\right)$.

Figure 16: Comparison between a kinematic approach and the micro-mechanical model presented for model B (-a: $\vartheta=0^{\circ}$; -b: $\vartheta=22.5^{\circ}$; -c: $\vartheta=45^{\circ}$.). 


\section{LIST OF TABLES:}

Table I: Linear elastic properties for mortar and units.

Table II: Mechanical characteristics for the example of Figure 8.

Table III: Geometry of the elementary cell for the example of Figure 8.

Table IV: Mechanical characteristics for Page biaxial tests.

Table V: Mechanical characteristics adopted for mortar joints of Raijmakers and Vermeltfoort. 


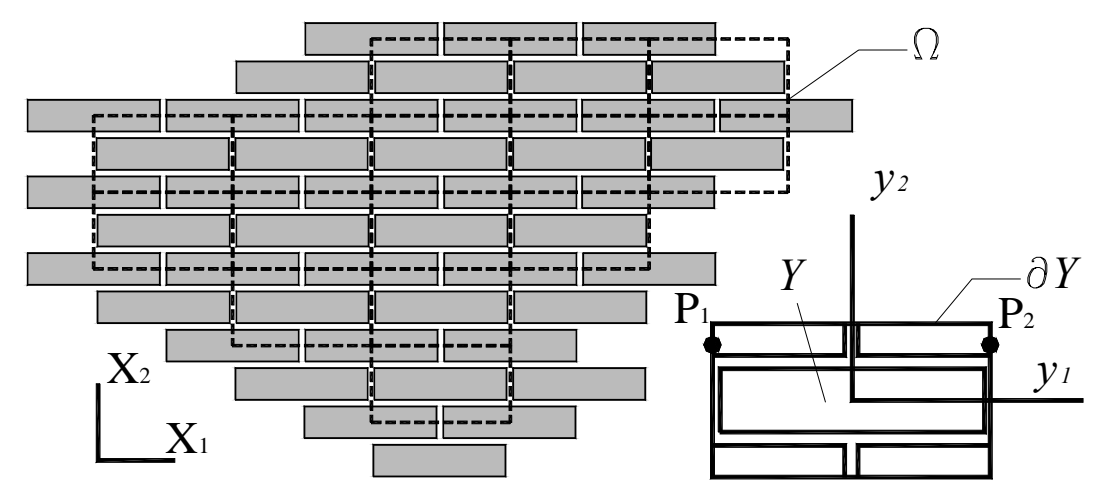

Figure 1 


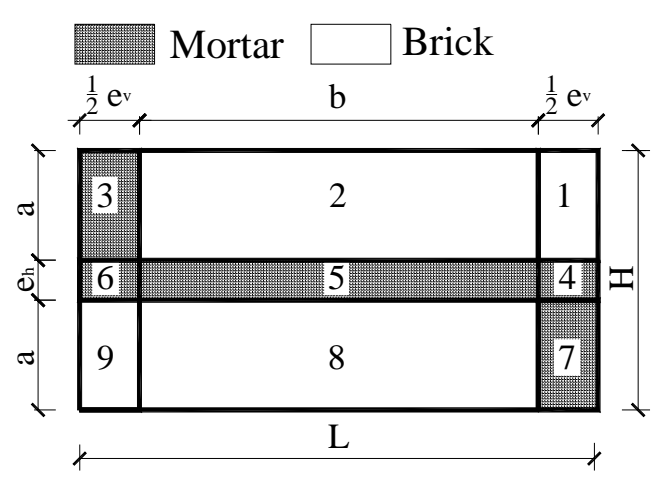

\begin{tabular}{|c|c|c|c|c|c|}
\hline 12 & 11 & 10 & & 2 & 1 \\
\hline 15 & 14 & 13 & & 5 & 4 \\
\hline 18 & 17 & 16 & 9 & 8 & 7 \\
\hline 30 & 29 & 28 & 21 & 20 & 19 \\
\hline 33 & 32 & 31 & 24 & 23 & 22 \\
\hline 36 & 35 & 34 & 27 & 26 & 25 \\
\hline
\end{tabular}

Figure 2 

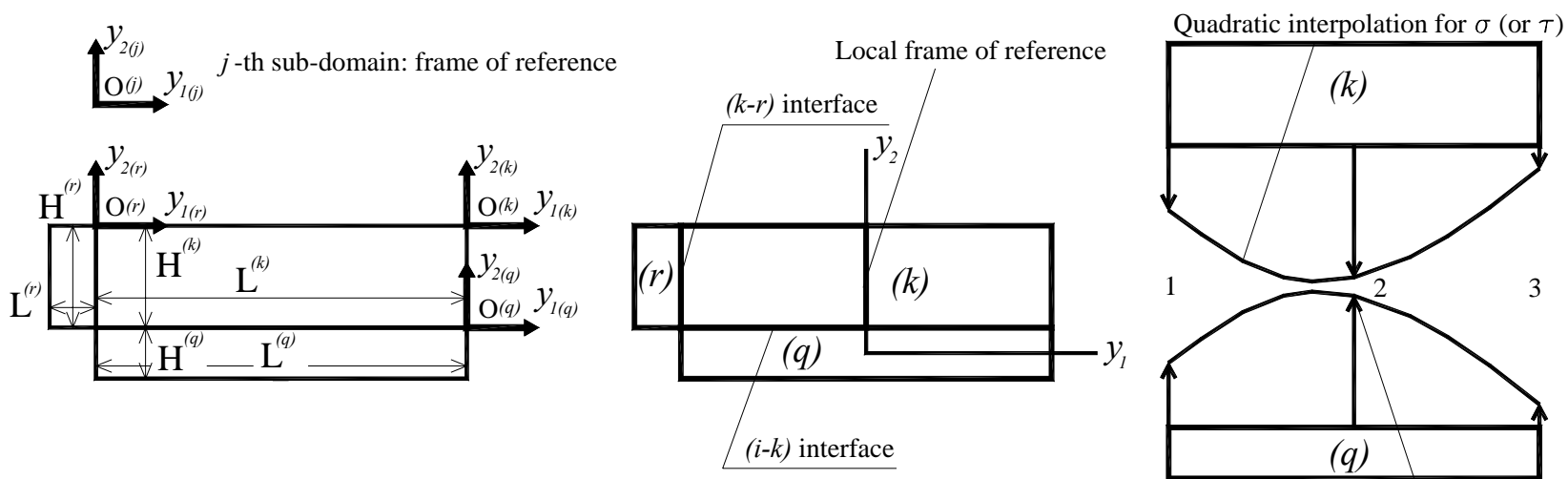

Equilibrium to check in 3 points

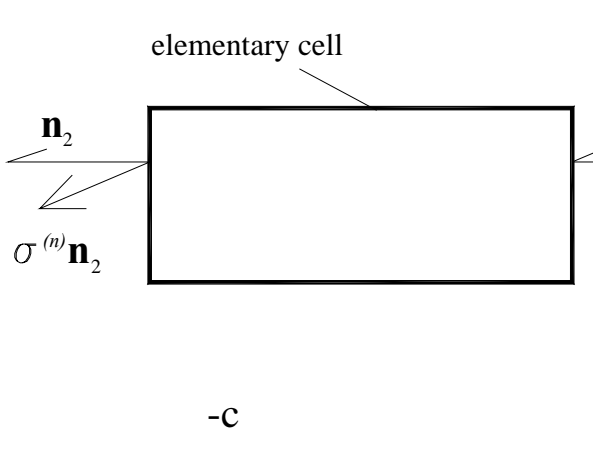

$\sigma^{(m)} \mathbf{n}_{1}$

$\mathbf{n}_{1}$

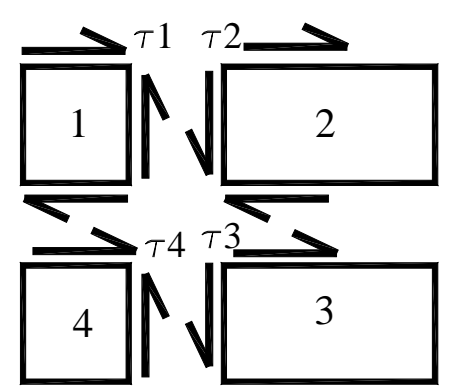

$-d$

Figure 3 

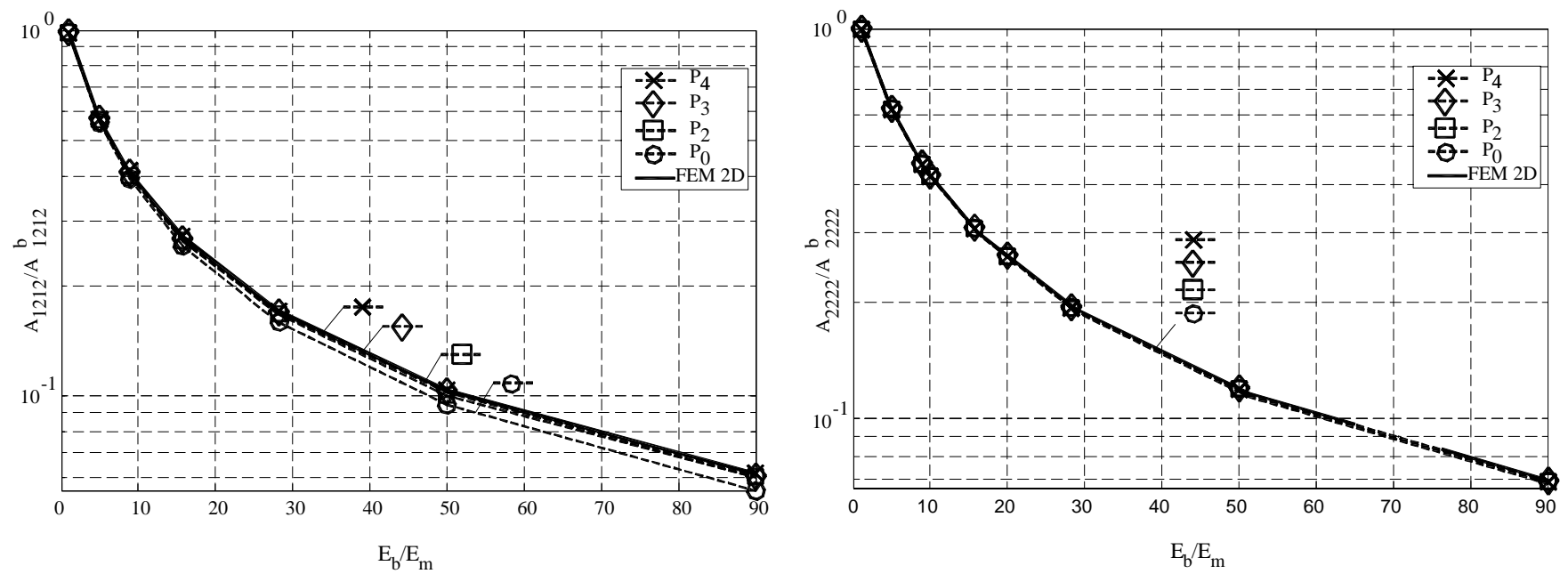

$-a$

$-b$
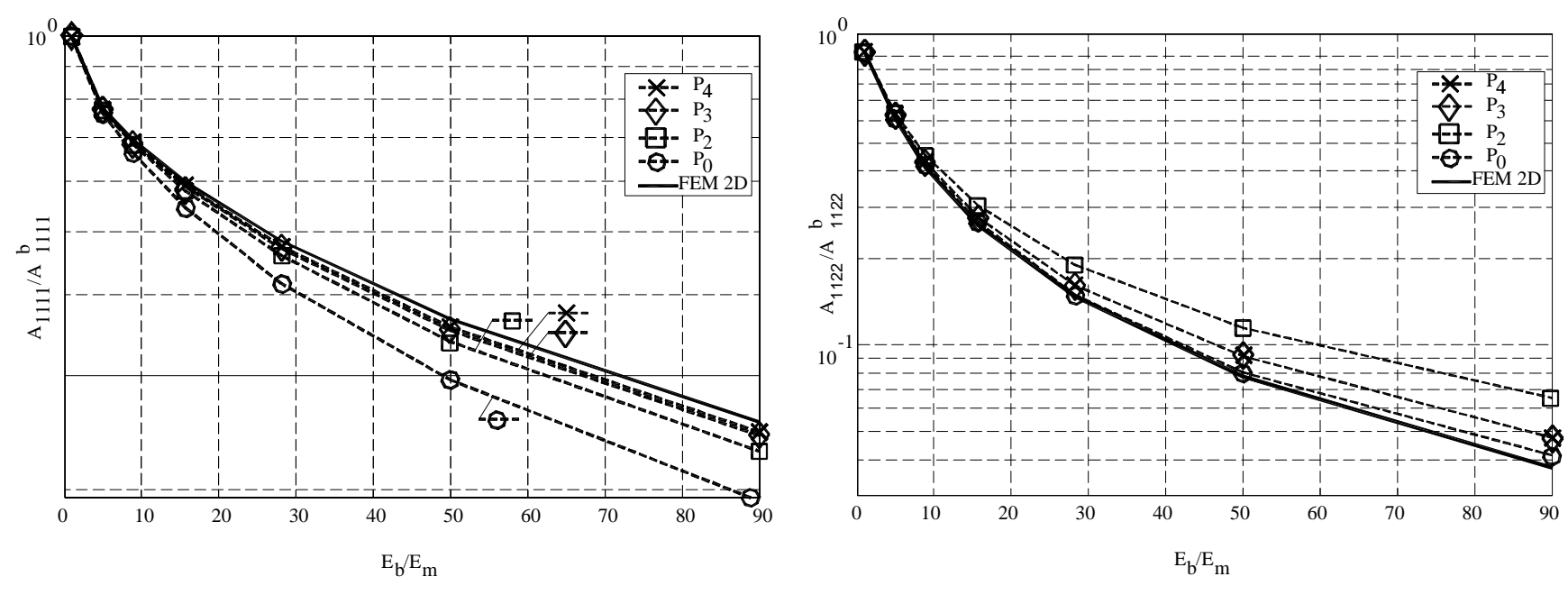

$-d$

Figure 4 


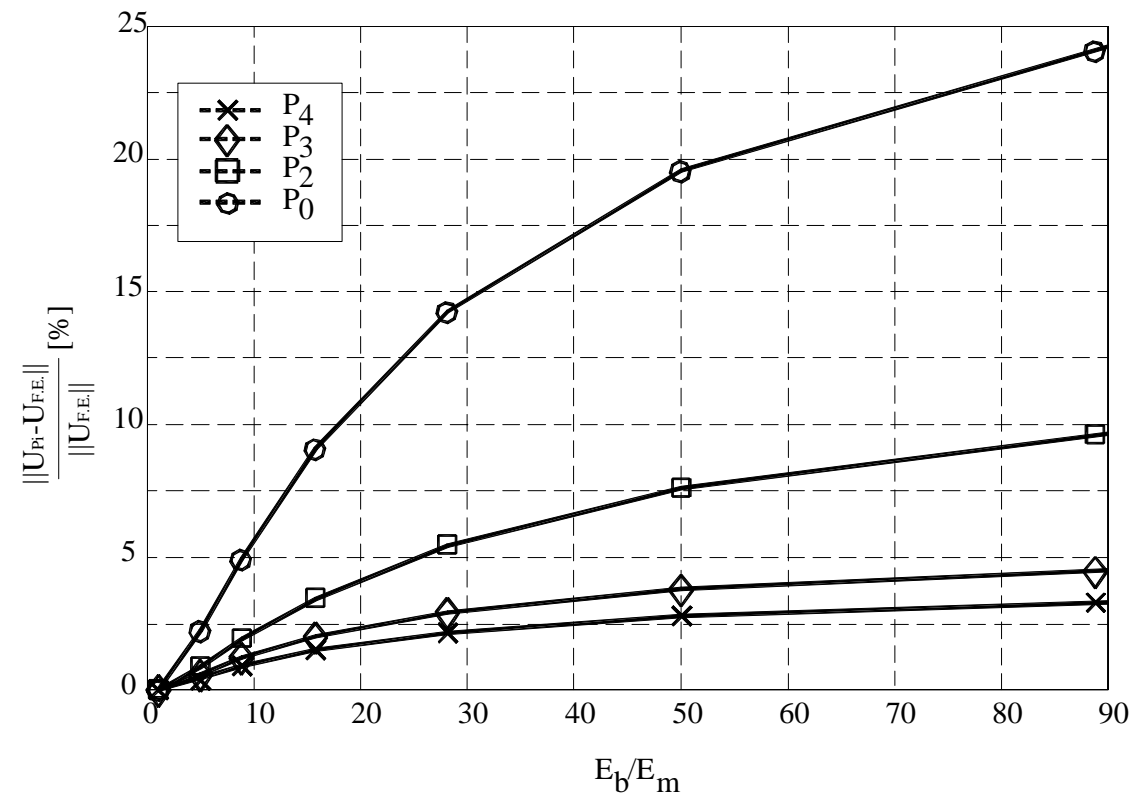

Figure 5 


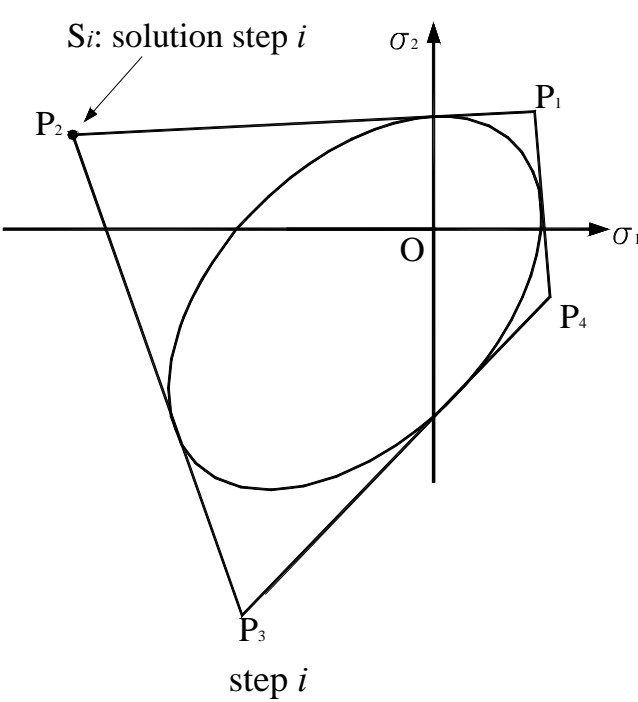

$-a$

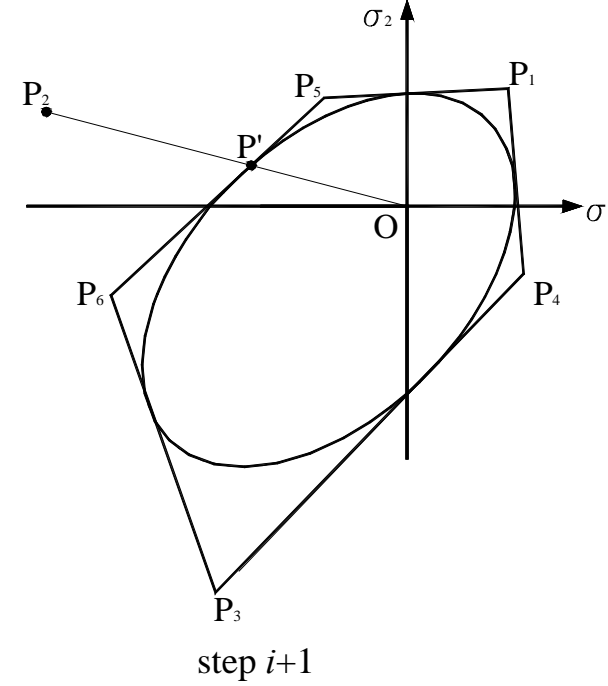

-b

Figure 6 


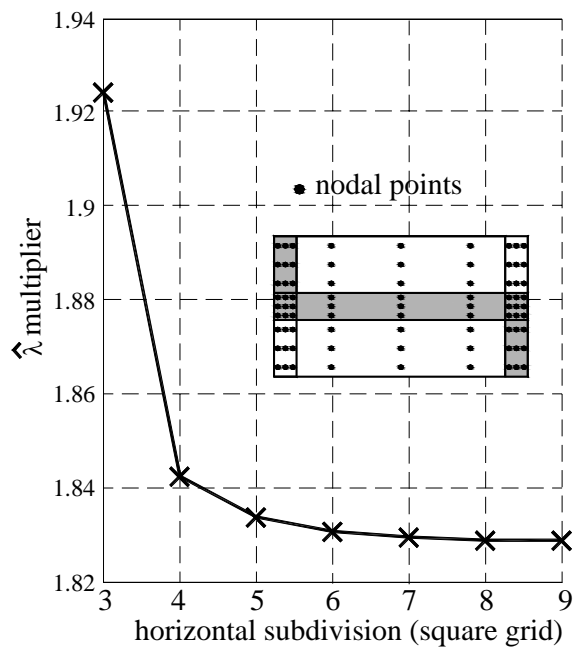

Figure 7 


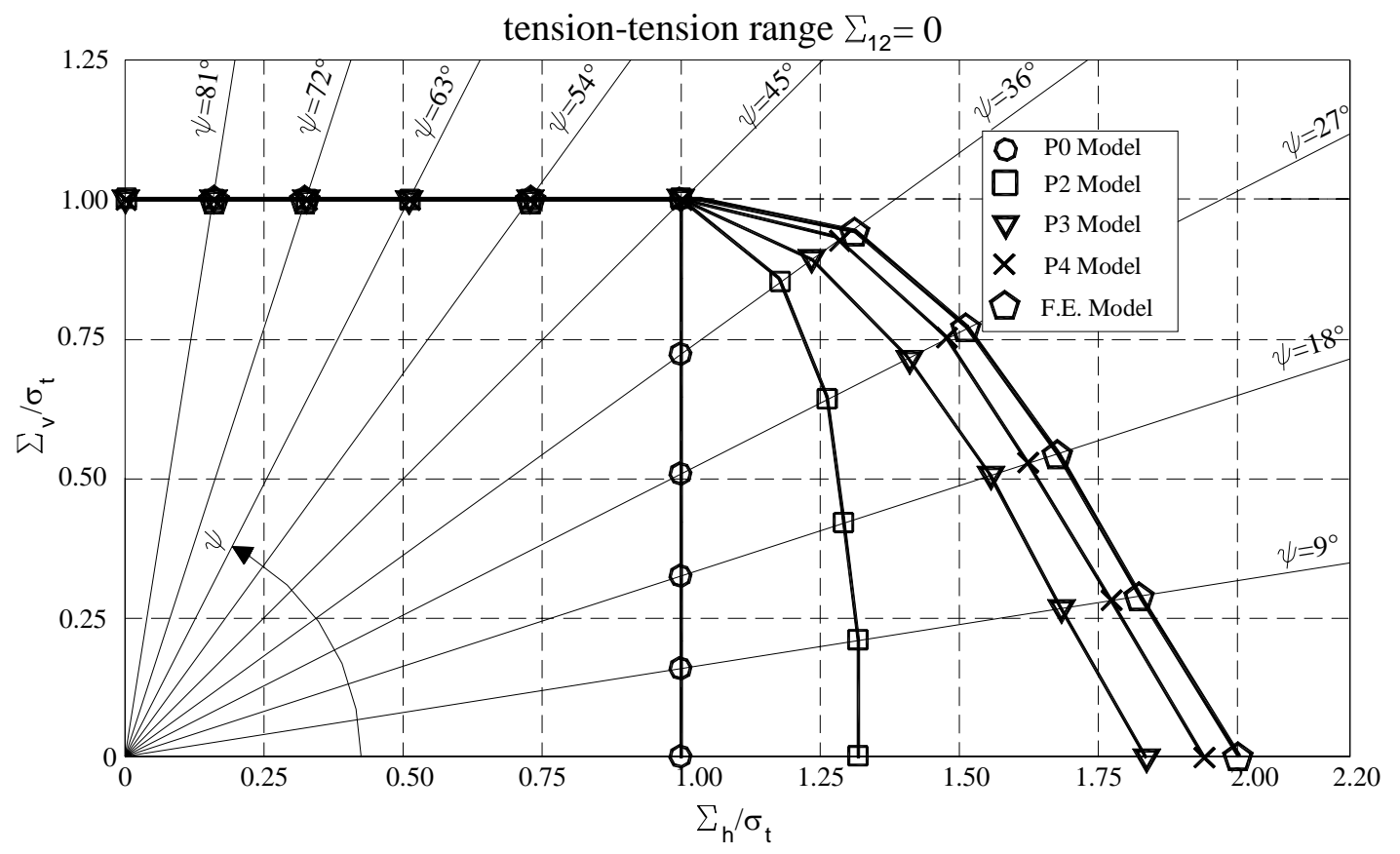

Figure 8 


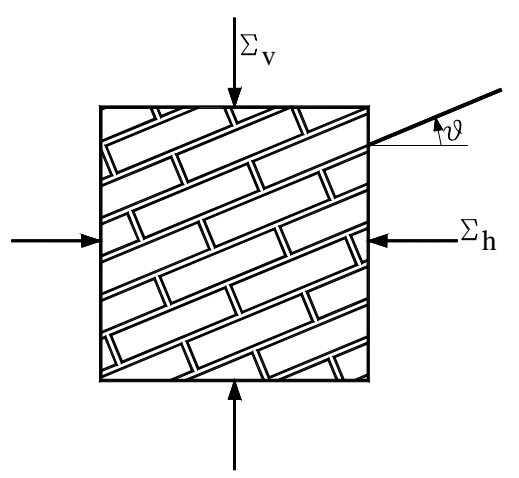

Figure 9 


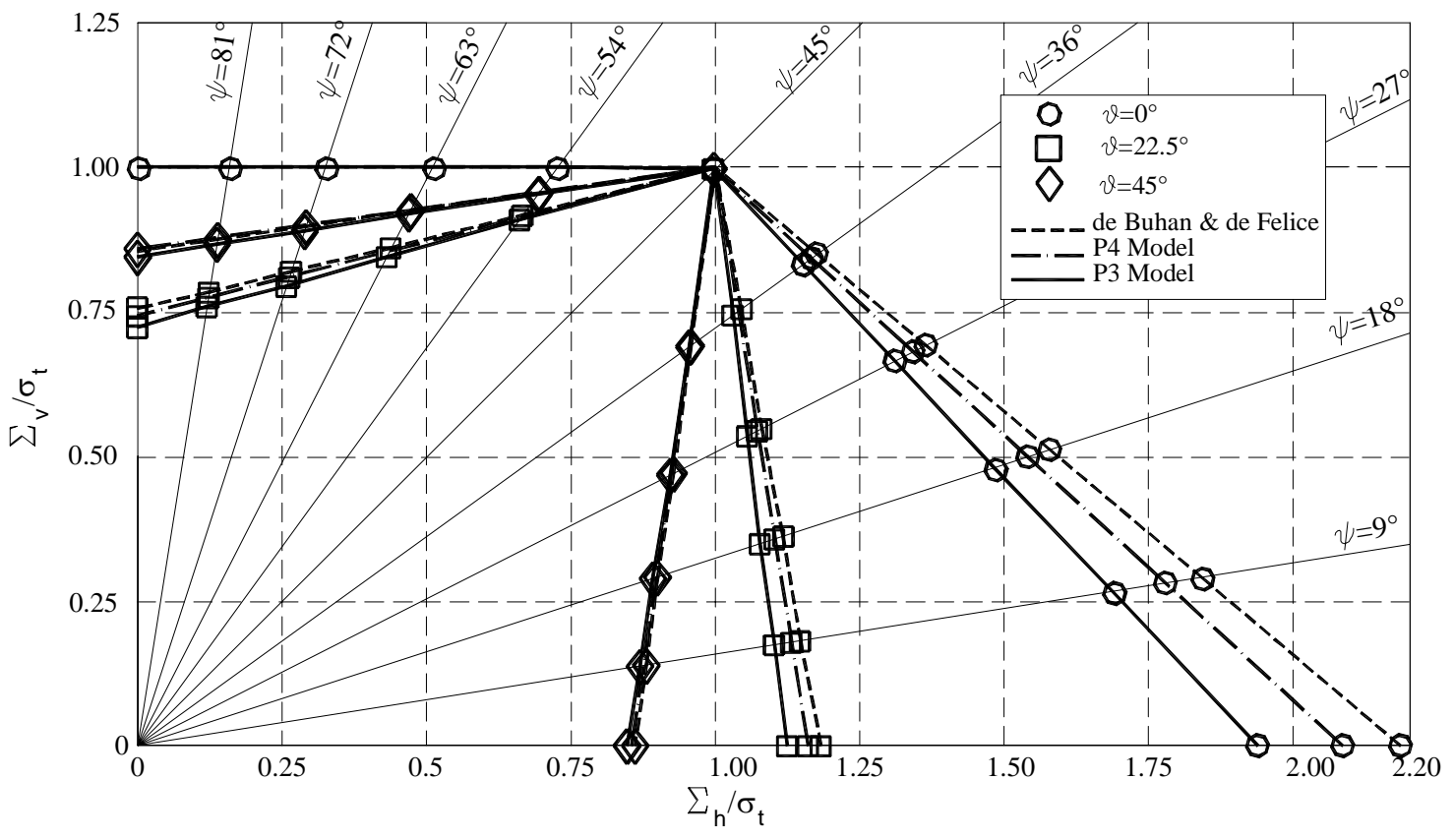

Figure 10 


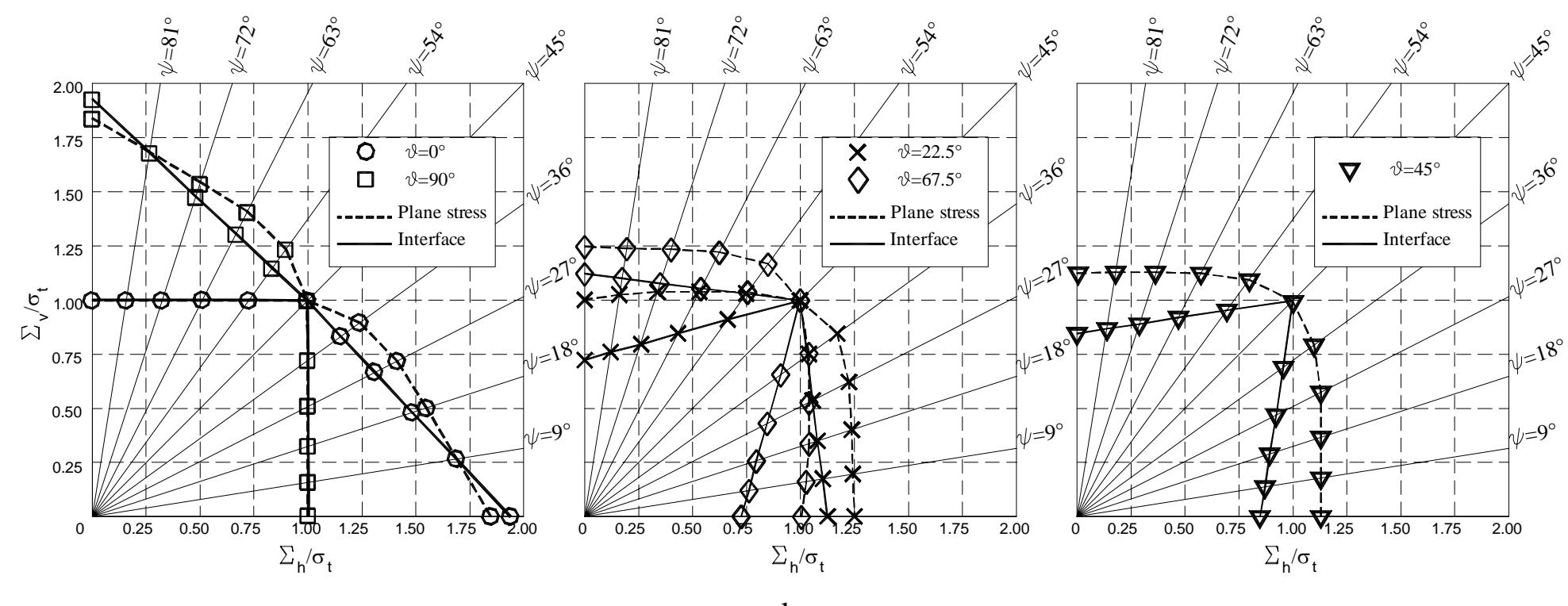

$-a-$

-b-

Figure 11 


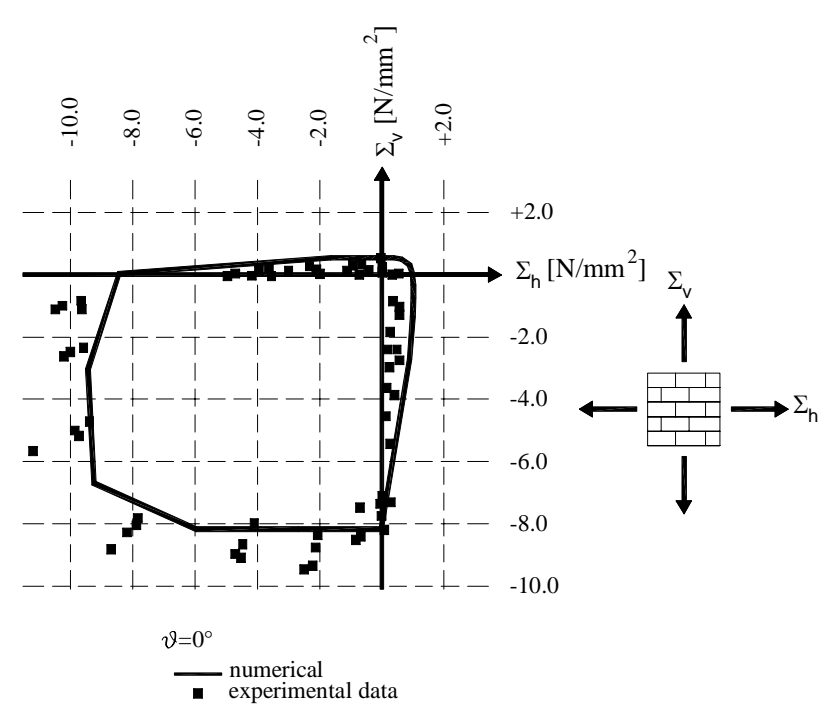

$-a$

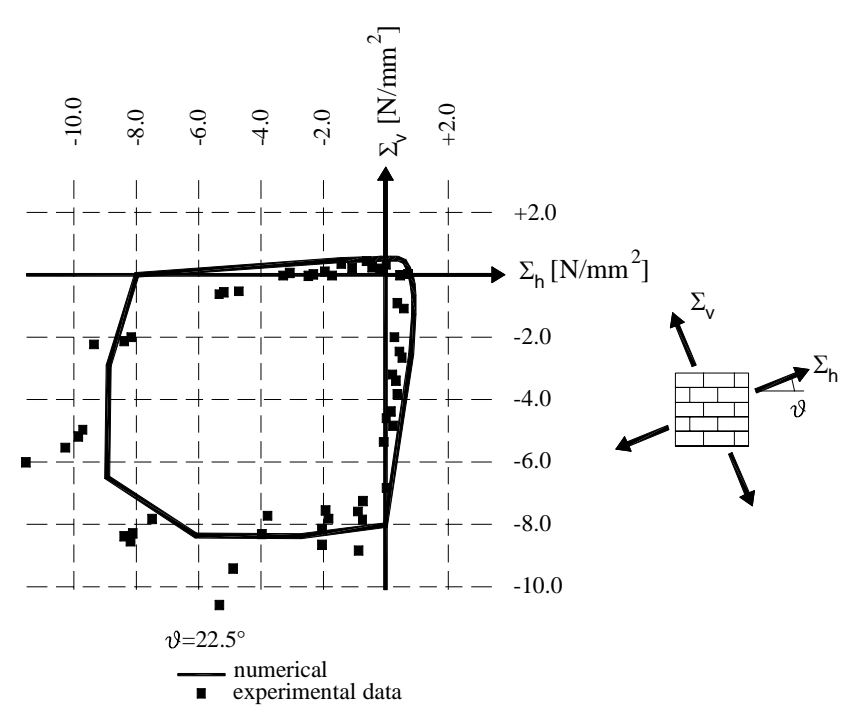

-b

Figure 12

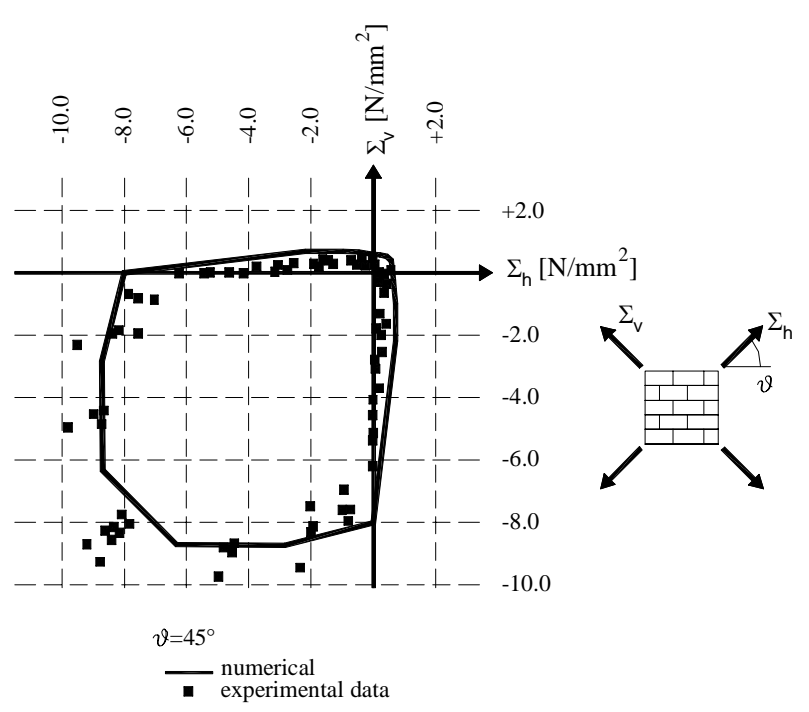

$-C$ 


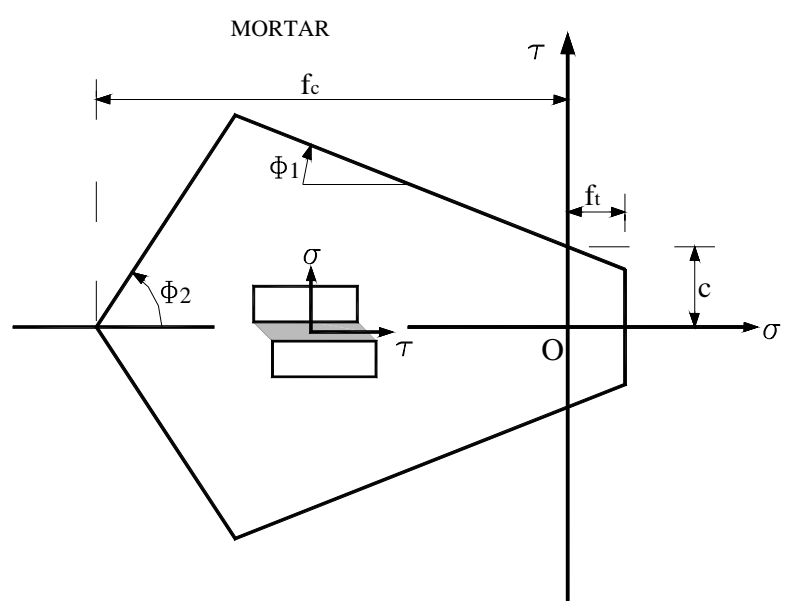

Figure 13 


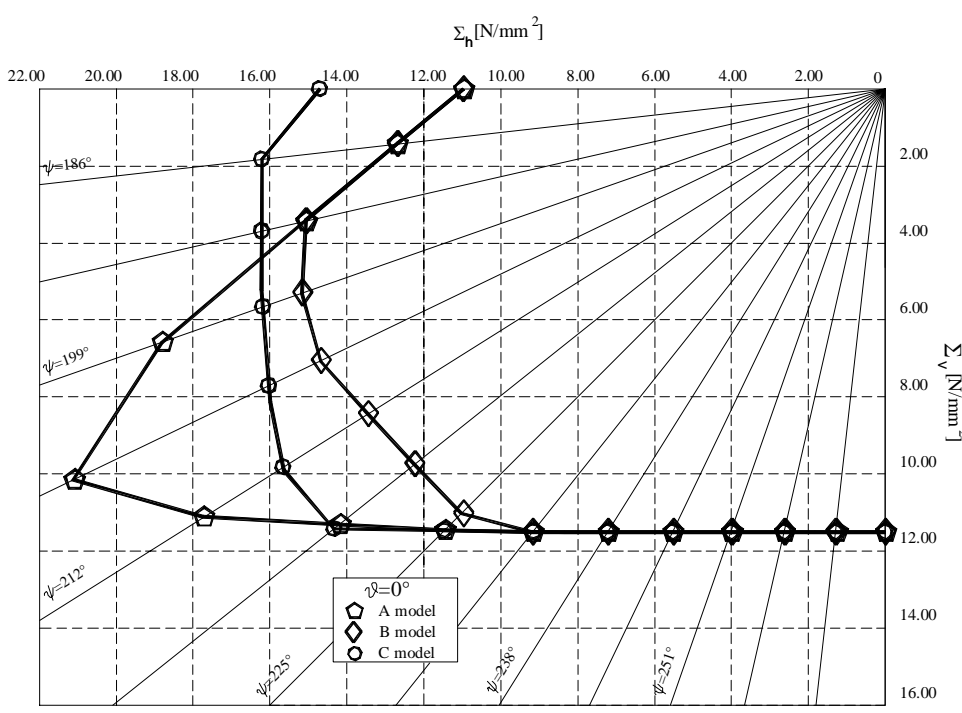

-a
$\Sigma_{\mathrm{h}}\left[\mathrm{N} / \mathrm{mm}^{2}\right]$

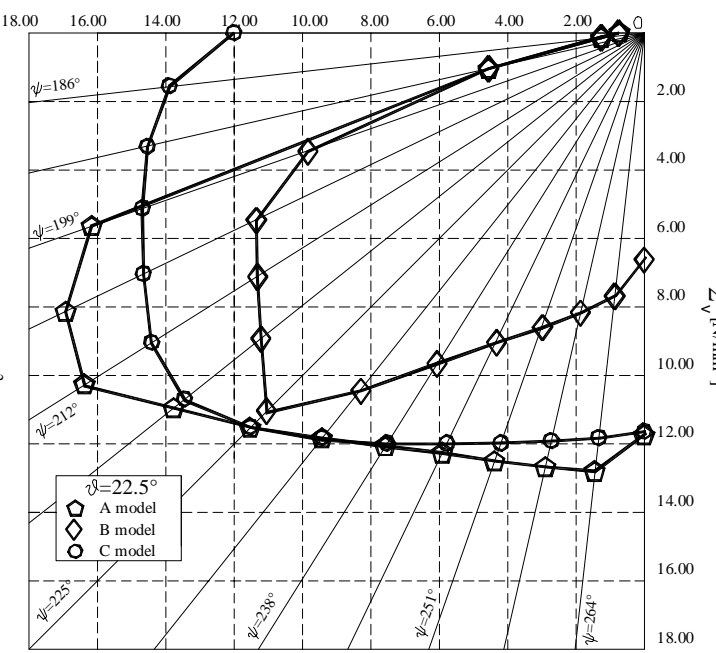

-b

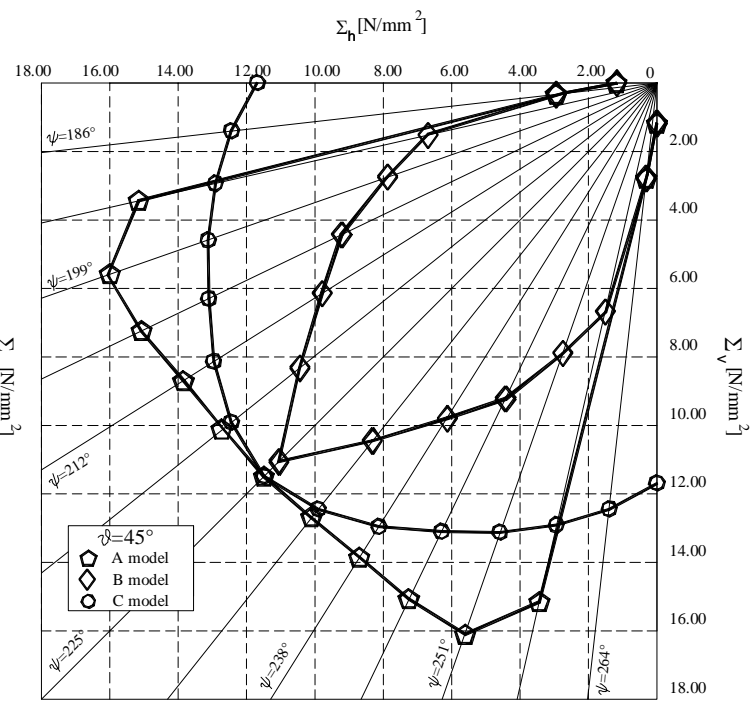

$-\mathrm{C}$

Figure 14 
$\Sigma_{\mathrm{h}}\left[\mathrm{N} / \mathrm{mm}^{2}\right]$

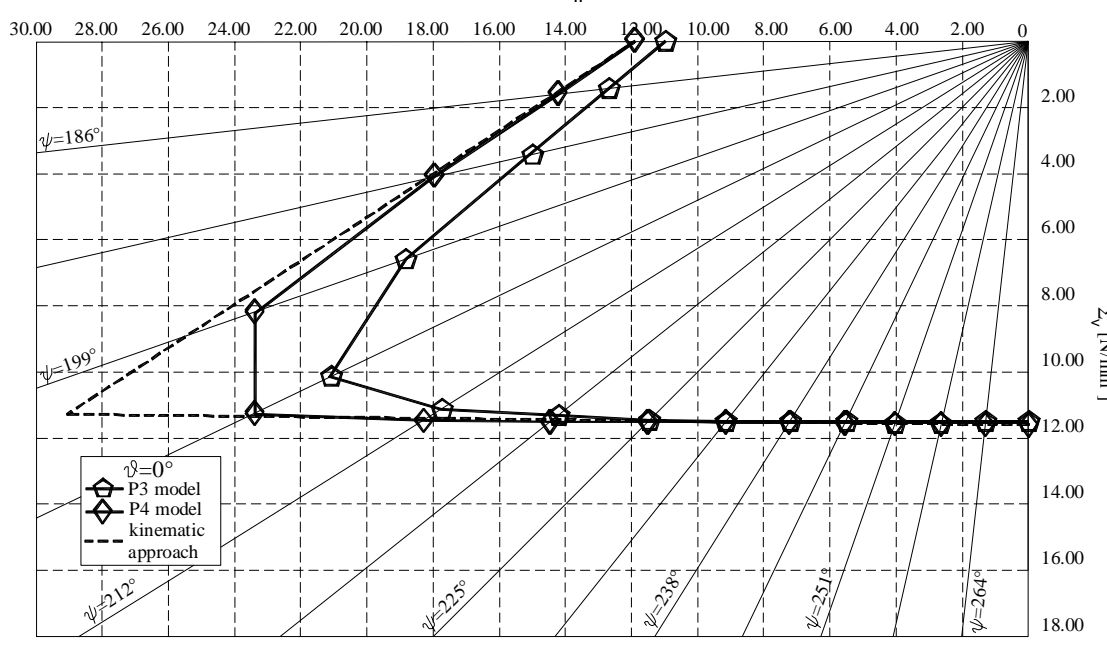

$-a$
$\Sigma_{\mathrm{h}}\left[\mathrm{N} / \mathrm{mm}^{2}\right]$

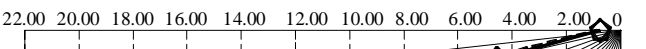

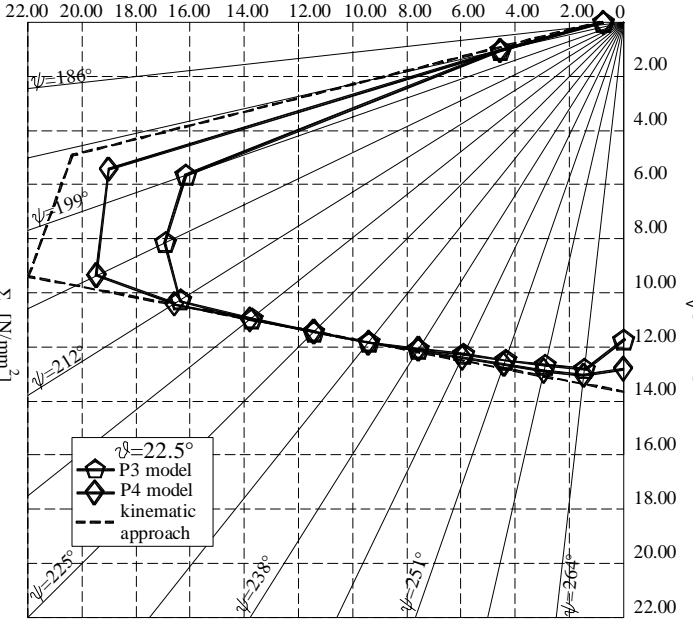

$-b$

Figure 15
$\Sigma_{\mathrm{h}}\left[\mathrm{N} / \mathrm{mm}^{2}\right]$

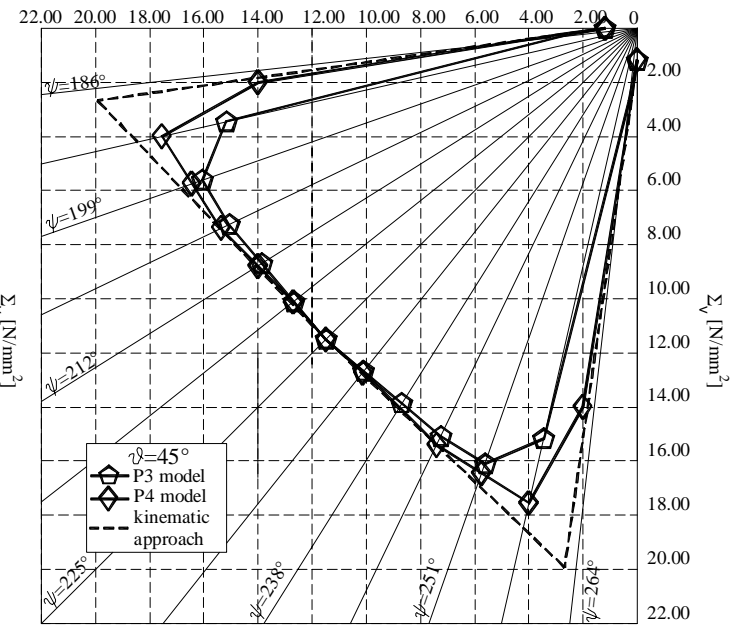

$-C$ 

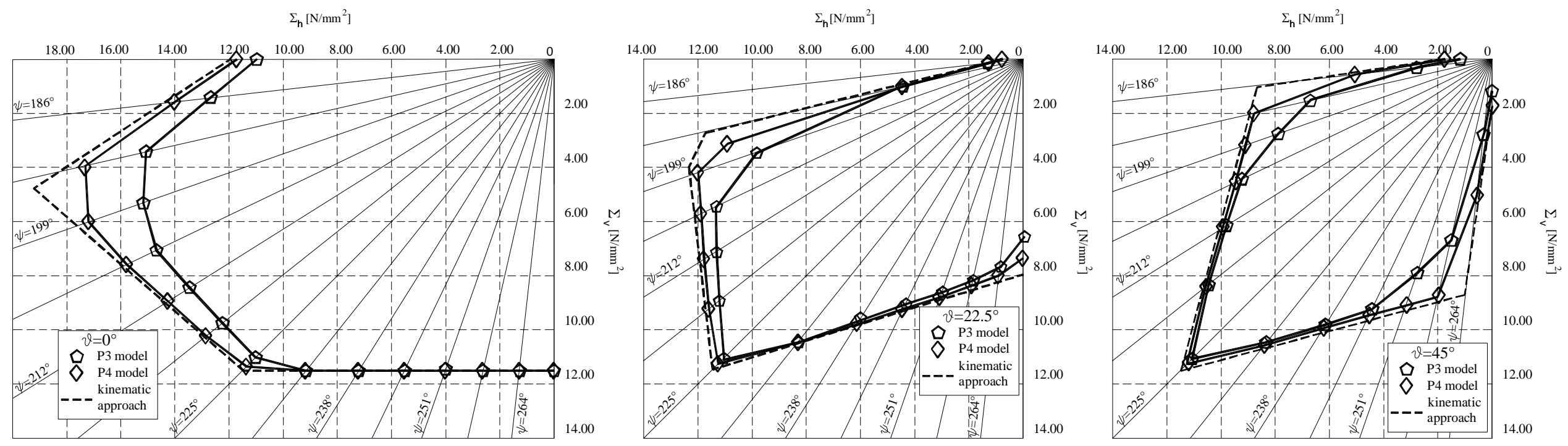

$-a$

-b

$-\mathrm{C}$

Figure 16

44 


\begin{tabular}{c} 
unit \\
\hline mortar \\
\hline
\end{tabular}

$\mathrm{E}\left[\mathrm{N} / \mathrm{mm}^{2}\right]$

Young modulus

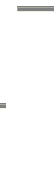

11000

2200 v

Poisson ratio

0.2

0.25

Table I 
Frictional angle ( $\Phi$ ) Cohesion (c)

\begin{tabular}{ccc}
$\sigma_{t}=\frac{2 c \cos (\Phi)}{1+\sin (\Phi)}$ & $\sigma_{c}=\frac{2 c \cos (\Phi)}{1-\sin (\Phi)}$ & $\begin{array}{r}\text { Infinitely } \\
\text { resistant }\end{array}$ \\
\hline
\end{tabular}

Table II 


\begin{tabular}{cc}
$\frac{b[\mathrm{~mm}]}{52.5}$ & $\mathrm{a}[\mathrm{mm}]$ \\
\hline $\begin{array}{c}\mathrm{e}_{\mathrm{h}}[\mathrm{mm}] \\
10\end{array}$ & $\begin{array}{c}17.5 \\
\mathrm{e}_{\mathrm{v}}[\mathrm{mm}]\end{array}$ \\
\cline { 1 - 2 } & $=\mathrm{e}_{\mathrm{h}}$ \\
\hline
\end{tabular}

Table III 
Mortar

\begin{tabular}{|c|c|}
\hline Compressive Strength & $\begin{array}{c}\text { Compressive/Tensile } \\
\text { strength ratio }\end{array}$ \\
\hline$\sigma_{c}=8.01\left[\mathrm{~N} / \mathrm{mm}^{2}\right]$ & $\sigma_{\mathrm{c}} / \sigma_{\mathrm{t}}=15.7$ \\
\hline
\end{tabular}

Unit

\begin{tabular}{|c|c|}
\hline Compressive Strength & $\begin{array}{c}\text { Compressive/Tensile } \\
\text { strength ratio }\end{array}$ \\
\hline$\sigma_{c}=10.1\left[\mathrm{~N} / \mathrm{mm}^{2}\right]$ & $\sigma_{c} / \sigma_{t}=6$ \\
\hline
\end{tabular}

Table IV 
Model A

\begin{tabular}{|c|c|c|c|c|c|}
\hline $\mathrm{f}_{\mathrm{t}}\left(\mathrm{c}=1.4 \mathrm{f}_{\mathrm{t}}\right)$ & $\mathrm{f}_{\mathrm{c}}$ & $\mathrm{f}_{\mathrm{t}}\left(\mathrm{c}=1.4 \mathrm{f}_{\mathrm{t}}\right)$ & $\mathrm{f}_{\mathrm{c}}$ & $\sigma_{c}$ & $\Phi$ \\
\hline $\begin{array}{c}0.16 \\
{\left[\mathrm{~N} / \mathrm{mm}^{2}\right]}\end{array}$ & $\begin{array}{c}11.5 \\
{\left[\mathrm{~N} / \mathrm{mm}^{2}\right]}\end{array}$ & $\begin{array}{c}0.16 \\
{\left[\mathrm{~N} / \mathrm{mm}^{2}\right]}\end{array}$ & $\begin{array}{c}11.5 \\
{\left[\mathrm{~N} / \mathrm{mm}^{2}\right]}\end{array}$ & $\begin{array}{c}11.5 \\
{\left[\mathrm{~N} / \mathrm{mm}^{2}\right]}\end{array}$ & $37^{\circ}$ \\
\hline$\Phi_{1}$ & $\Phi_{2}$ & $\Phi_{1}$ & $\Phi_{2}$ & $c=\sigma \underline{1-\sin (\Phi)}$ & $\sigma=\frac{2 c \cos (\Phi)}{}$ \\
\hline $37^{\circ}$ & $90^{\circ}$ & $37^{\circ}$ & $30^{\circ}$ & $2 \cos (\Phi)$ & $1+\sin (\Psi)$ \\
\hline
\end{tabular}

Table V

Model C
Model B

\section{Table V}

